\title{
1 Distinct temporal difference error signals in dopamine axons in three regions \\ 2 of the striatum in a decision-making task
}

3

4 Iku Tsutsui-Kimura ${ }^{1}$, Hideyuki Matsumoto ${ }^{1,2}$, Naoshige Uchida $^{1}$ and Mitsuko Watabe-Uchida ${ }^{1,3, *}$ 5

6

9 Affiliations:

$10{ }^{1}$ Department of Molecular and Cellular Biology, Center for Brain Science, Harvard University,

11 Cambridge, MA 02138, USA

$12{ }^{2}$ Department of Physiology, Osaka City University Graduate School of Medicine, Osaka, 545-8585, Japan

$13{ }^{3}$ Lead Contact

14

$15 *$ Correspondence: mitsuko@mcb.harvard.edu (M.W.-U.)

16

17 


\section{SUMMARY}

20

21 Different regions of the striatum regulate different types of behavior. However, how dopamine

22 signals differ across striatal regions and how dopamine regulates different behaviors remain

23 unclear. Here, we compared dopamine axon activity in the ventral, dorsomedial, and dorsolateral

24 striatum, while mice performed in a perceptual and value-based decision task. Surprisingly,

25 dopamine axon activity was similar across all three areas. At a glance, the activity multiplexed

26 different variables such as stimulus-associated values, confidence and reward feedback at

27 different phases of the task. Our modeling demonstrates, however, that these modulations can be

28 inclusively explained by moment-by-moment changes in the expected reward, i.e. the temporal

29 difference error. A major difference between these areas was the overall activity level of reward

30 responses: reward responses in dorsolateral striatum (DLS) were positively shifted, lacking

31 inhibitory responses to negative prediction error. Tenets of habit and skill can be explained by

32 this positively biased dopamine signal in DLS.

\section{Keywords}

37 dopamine, TD error, confidence, value, striatum, choice, feedback 


\section{INTRODUCTION}

48 Flexibility in behavior relies critically on an animal's ability to alter behavior based on past experiences. In particular, the behavior of the animal is greatly shaped by the consequences of specific actions - whether a previous action led to positive or negative experiences. One of the fundamental questions in neuroscience is how animals learn from rewards and punishments.

A neurotransmitter, dopamine, is thought to be a key regulator of learning from rewards and punishments (Hart et al., 2014; Montague et al., 1996; Schultz et al., 1997). Neurons that release dopamine (hereafter, dopamine neurons) are located mainly in the ventral tegmental area (VTA) and substantia nigra pars compacta $(\mathrm{SNc})$. These neurons send their axons to various regions

57 including the striatum, neocortex, and amygdala (Menegas et al., 2015; Yetnikoff et al., 2014).

58 The striatum, which receives the densest projection from VTA and SNc dopamine neurons, is thought to play particularly important roles in learning from rewards and punishments (Lloyd and Dayan, 2016; O’Doherty et al., 2004). However, what information dopamine neurons convey to the striatum, and how dopamine regulates behavior through its projections to the striatum remain elusive.

A large body of experimental and theoretical studies have suggested that dopamine neurons signal reward prediction errors (RPEs) - the discrepancy between actual and predicted rewards (Bayer and Glimcher, 2005; Cohen et al., 2012; Hart et al., 2014; Schultz et al., 1997). In particular, the activity of dopamine neurons resembles a specific type of prediction error, called temporal difference RPE (TD error) (Montague et al., 1996; Schultz et al., 1997; Sutton, 1988; Sutton and Barto, 1987). Although it was widely assumed that dopamine neurons broadcast homogeneous RPEs to a swath of dopamine-recipient areas, recent findings indicated that

71 dopamine signals are more diverse than previously thought (Brown et al., 2011; Kim et al., 2015;

72 Matsumoto and Hikosaka, 2009; Menegas et al., 2017, 2018; Parker et al., 2016). For one, recent

73 studies have demonstrated that a transient ("phasic") activation of dopamine neurons occurs near

74 the onset of a large movement (e.g. locomotion), regardless of whether these movements are

75 immediately followed by a reward (Howe and Dombeck, 2016; da Silva et al., 2018). These

76 phasic activations at movement onsets have been observed in the somatic spiking activity in the 
77 SNc (da Silva et al., 2018) as well as the axonal activity in the dorsal striatum (Howe and

78 Dombeck, 2016). Another study showed that dopamine axons in the dorsomedial striatum

79 (DMS) are activated when the animal makes a contralateral orienting movement in a decision-

80 making task (Parker et al., 2016). Other studies have also found that dopamine axons in the

81 posterior or ventromedial parts of the striatum are activated by aversive or threat-related stimuli

82 (de Jong et al., 2019; Menegas et al., 2017). An emerging view is that dopamine neurons

83 projecting to different parts of the striatum convey distinct signals and support different

84 functions (Cox and Witten, 2019).

Previous studies have shown that different parts of the striatum control distinct types of reward-

87 oriented behaviors (Dayan and Berridge, 2014; Graybiel, 2008; Malvaez and Wassum, 2018;

88 Rangel et al., 2008). First, the ventral striatum (VS) has often been associated with Pavlovian

89 behaviors, where the expectation of reward triggers relatively pre-programmed behaviors

90 (approaching, consummatory behaviors etc.) (Dayan and Berridge, 2014). Psychological studies

91 suggest that these behaviors are driven by stimulus-outcome associations (Kamin, 1969; Pearce

92 and Hall, 1980; Rescorla and Wagner, 1972). Consistent with this idea, previous experiments

93 have shown that dopamine in VS conveys canonical RPE signals (Menegas et al., 2017; Parker et

94 al., 2016), and support learning of values associated with specific stimuli (Clark et al., 2012). In

95 contrast, the dorsal part of the striatum has been linked to instrumental behaviors, where animals

96 acquire an arbitrary action that leads to a reward (Montague et al., 1996; Suri and Schultz, 1999).

97 Instrumental behaviors are further divided into two distinct types: goal-directed and habit

98 (Dickinson and Weiskrantz, 1985). Goal-directed behaviors are "flexible" reward-oriented

99 behaviors that are sensitive to a causal relationship ("contingency") between action and outcome, 100 and can quickly adapt to changes in the value of the outcome (Balleine and Dickinson, 1998).

101 After repetition of a goal-directed behavior, the behavior can become a habit which is

102 characterized by insensitivity to changes in the outcome value (e.g. devaluation) (Balleine and

103 O'Doherty, 2010). According to psychological theories, goal-directed and habitual behaviors are

104 supported by distinct internal representations: action-outcome and stimulus-response

105 associations, respectively (Balleine and O'Doherty, 2010). Lesion studies have indicated that

106 goal-directed behaviors and habit are controlled by DMS and the dorsolateral striatum (DLS),

107 respectively (Yin et al., 2004, 2005). 
109 Instrumental behaviors are shaped by reward, and it is generally thought that dopamine is involved in their acquisition (Gerfen and Surmeier, 2011; Montague et al., 1996; Schultz et al.,

111 1997). However, how dopamine is involved in distinct types of instrumental behaviors remain

112 unknown. A prevailing view in the field is that habit is controlled by "model-free" reinforcement 113 learning, while goal-directed behaviors are controlled by "model-based" mechanisms (Daw et

114 al., 2005; Dolan and Dayan, 2013; Rangel et al., 2008). In this framework, habitual behaviors are 115 driven by "cached" values associated with specific actions (action values) which animals learn 116 through direct experiences via dopamine RPEs. In contrast, goal-directed behaviors are 117 controlled by a "model-based" mechanism whereby action values are computed by mentally 118 simulating which sequence of actions lead to which outcome using a relatively abstract 119 representation (model) of the world. Model-based behaviors are more flexible compared to 120 model-free behaviors because a model-based mental simulation may allow the animal to 121 compute values in novel or changing circumstances. Although these ideas account for the 122 relative inflexibility of habit over model-based, goal-directed behaviors, they do not necessarily 123 explain the most fundamental property of habit, that is, its insensitivity to changes in outcome, as 124 cached values can still be sensitive to RPEs when the actual outcome violates expectation, 125 posing a fundamental limit in this framework (Dezfouli and Balleine, 2012; Miller et al., 2019).

126 Furthermore, the idea that habits are supported by action value representations does not 127 necessarily match with the long-held view of habit based on stimulus-response associations.

Until recently an implicit assumption across many studies was that dopamine neurons broadcast the same teaching signals throughout the striatum to support different kinds of learning (Rangel et al., 2008; Samejima and Doya, 2007). However, as mentioned before, more recent studies revealed different dopamine signals across striatal regions, raising the possibility that different

133 striatal regions receive distinct teaching signals. In any case, few studies have directly examined 134 the nature of RPE signals across striatal regions in instrumental behaviors, in particular, between DLS and other regions. As a result, it remains unclear whether different striatal regions receive 136 distinct dopamine signals during instrumental behaviors. Are dopamine signals in particular 137 areas dominated by movement-related signals? Are dopamine signals in these areas still consistent with RPEs or are they fundamentally distinct? How are they different? Characterizing 
dopamine signals in different regions is a critical step toward understanding how dopamine may regulate distinct types of behavior.

In the present study, we sought to characterize dopamine signals in different striatal regions (VS, DMS and DLS) during instrumental behaviors. We used a task involving both perceptual and value-based decisions in freely-moving mice - a task that is similar to those previously used to probe various important variables in the brain such as values, biases (Rorie et al., 2010; Wang et al., 2013), confidence (Hirokawa et al., 2019; Kepecs et al., 2008), belief states (Lak et al., 2017), and response vigor (Wang et al., 2013). In this task, the animal goes through various movements and mental processes - self-initiating a trial, collecting sensory evidence, integrating the sensory evidence with reward information, making a decision, initiating a choice movement, committing to an option and waiting for reward, receiving an outcome of reward or no reward, and adjusting internal representations for future performance using RPEs and confidence. Compared to Pavlovian tasks, which have been more commonly used to examine dopamine RPEs, the present task has various factors with which to contrast dopamine signals between different areas.

Contrary to our initial hypothesis, dopamine signals in all three areas showed similar dynamics, going up and down in a manner consistent with TD errors, reflecting moment-by-moment changes in the expected future reward (i.e. state values). Notably, although we observed correlates of accuracy and confidence in dopamine signals, consistent with previous studies (Engelhard et al., 2019; Lak et al., 2017), the appearance of these variables was timing- and trial type-specific. In stark contrast, our modeling demonstrate that these apparently diverse dopamine signals can be inclusively explained by a single variable - TD error, that is moment-by-moment changes in the expected reward in each trial. In addition, we found consistent differences between these areas. For instance, DMS dopamine signals were modulated by contralateral orienting movements, as reported previously (Parker et al., 2016). Furthermore, DLS dopamine signals, while following TD error dynamics, were overall more positive, compared to other regions. Based on these findings, we present novel models of how these distinct dopamine signals may give rise to distinct types of behavior such as flexible versus habitual behaviors. 


\section{RESULTS}

Mice were first trained in a perceptual decision-making task using olfactory stimuli (Figure 1) mixed with different ratios (Figure 1A). Mice were required to initiate a trial by poking their nose into the central odor port, which triggered a delivery of an odor mixture. Mice were then required to move to the left or right water port depending on which odor was dominant in the presented mixture. Odor-water side (left or right) rule was held constant throughout training and recording in each animal. In order to minimize temporal overlaps between different trial events and underlying brain processes, we introduced a minimum time required to stay in the odor port (for $1 \mathrm{~s}$ before exiting the odor port) and in the water port (for $1 \mathrm{~s}$ ) to receive a water reward.

After mice learned the task, the water amounts at the left and right water ports were manipulated (Lak et al., 2017; Rorie et al., 2010; Wang et al., 2013) in a probabilistic manner. In our task, one of the reward ports was associated with a big or medium size of water (BIG side) while another side was associated with a small or medium size of water (SMALL side) (Figure 1A). In a daily session, there were two blocks of trials, the first with equal-sized water and the second with different distributions of water sizes on the two sides (BIG versus SMALL side). The reward ports for BIG or SMALL conditions stayed unchanged within a session, and were randomly chosen for each session. In each reward port (BIG or SMALL side), which of the two reward sizes was delivered was randomly assigned in each trial. Note that the medium-sized reward is

194 delivered with the probability of 0.5 for every correct choice at either side. This design was used to facilitate our ability to characterize RPE-related responses even after mice were well trained (Tian et al., 2016). First, the responses to the medium sized-reward allowed us to characterize

197 how "reward expectation" affects dopamine reward responses because we can examine how different levels of expectation, associated with the BIG and SMALL side, affect dopamine responses to reward of the same (medium) amount. Conversely, for a given reward port, two 
sizes of reward allow us to characterize the effect of "actual reward" on dopamine responses, by comparing the responses when the actual reward was smaller versus larger than expected.

We first characterized the choice behavior by fitting a psychometric function (a logistic function). Compared to the block with equal-sized water, the psychometric curve was shifted laterally to the BIG side (Figure 1B, Figure S1). The fitted psychometric curves were laterally shifted whereas the slopes were not significantly different across blocks $(p=0.45)$ (Figure 1B).

207 We, therefore, quantified a choice bias as a lateral shift of the psychometric curve with a fixed slope in terms of the \% mixture of odors for each mouse (Figure 1C) (Wang et al., 2013). All the mice exhibited a choice bias toward the BIG side (22/22 animals). Because a "correct" choice (i.e. whether a reward is delivered or not) was determined solely by the stimulus in this task,

211 biasing their choices away from the 50/50 boundary inevitably lowers the choice accuracy (or

212 equivalently, the probability of reward). For ambiguous stimuli, however, mice could go for a

213 big reward, even sacrificing accuracy, in order to increase the long-term gain. Indeed, the observed biases approximated the optimal bias that maximizes total reward $(1.016 \pm 0.001$ times reward compared to no bias, mean \pm s.e.m, slightly less than the optimal bias that yields 1.022 times reward compared to no bias), rather than maximizing the accuracy (= reward probability, i.e. no bias) or solely minimizing the risk (the variance of reward amounts) (Figures 1D and 1E).

Previous studies have shown that animals shift their decision boundary even without reward amount manipulations in perceptual decision tasks (Lak et al., 2020a). These shifts occur on a trial-by-trial basis, following a win-stay strategy, choosing the same side when that side was

222 associated with reward in the previous trial, particularly when the stimulus was more ambiguous

223 (Lak et al., 2020a). In the current task design, however, the optimal bias is primarily determined

224 by the sizes of reward (more specifically, which side delivered a big or small reward) which

225 stays constant across trials within a session. To determine whether the animal adopted a short-

226 time scale updating or a more stable bias, we next examined how receipt of reward affected the

227 choice in the subsequent trials. To extract trial-by-trial updating, we compared the psychometric 228 curves 1 trial before $(n-1)$ and after $(n+1)$ the current trials $(n)$. This analysis was performed 229 separately for the rewarded side in the current (n) trials. We found that choice biases before and after a specific reward location were not significantly different in any trial types (Figure 1F), 
231 suggesting that trial-by-trial updating was minimum, contrary to a previous study (Lak et al.,

232 2020b). Instead, these results indicate that the mice adopted a relatively stable bias that lasts

233 longer than one trial.

234

235 Although we imposed a minimum time required to stay in the odor port, the mice showed

236 different reaction times (the duration between odor onset and odor port exit) across different trial

237 types (Figure 1G). First, reaction times were shorter when animals chose the BIG side compared

238 to the SMALL side in easy, but not difficult, trials. Second, reaction times were positively

239 correlated with the level of sensory evidence for choice (as determined by odor \% for the choice)

240 when mice chose the BIG side. However, this modulation was not evident when mice chose the

241 SMALL side.

242

\section{Overall activity pattern of dopamine axons in the striatum}

245 To monitor the activity of dopamine neurons in a projection specific manner, we recorded the

246 dopamine axon activity in the striatum using a calcium indicator, GCaMP7f (Dana et al., 2019)

247 with fiber fluorometry (Kudo et al., 1992) (fiber photometry) (Figure 2). We targeted a wide

248 range of the striatum including the relatively dorsal part of VS, DMS and DLS (Figure 2B).

249 Calcium signals were monitored from mice both before and after introducing water amount

250 manipulations ( $n=9,7,6$ mice, for VS, DMS, DLS).

252 The main analysis was performed using the calcium signals obtained in the presence of water

253 amount manipulations. To isolate responses that are time-locked to specific task events but with

254 potentially overlapping temporal dynamics, we first fitted dopamine axon activity in each animal

255 with a linear regression model using multiple temporal kernels (Park et al., 2014) with Lasso

256 regularization with 10-fold cross validation (Figure 2). We used kernels that extract stereotypical

257 time courses of activity locked to four different events: odor onset (odor), odor port exit

258 (movement), water port entry (choice commitment or "choice" for short), and reward delivery

259 (water) (Figures 2C-2F). 
261 The constructed model captured modulations of dopamine axon activity time-locked to different

262 events (Figure 2C). On average, the magnitude of the extracted odor-locked activity was

263 modulated by odor cues. Dopamine axons were more excited by a pure odor associated with the

264 BIG side than a pure odor associated with the SMALL side (Figures 2C and 2F). The movement-

265 locked activity was stronger for a movement toward the contra-lateral, compared to the ipsi-

266 lateral side, which was most evident in DMS (Parker et al., 2016) but much smaller in VS or

267 DLS (Figure 2E, \%Explained by movement). The choice-locked activity showed two types of

268 modulations (Figure 2C). First, it exhibited an inhibition in error trials at the time of reward (i.e.

269 when it has become clear that reward is not going to come). Second, dopamine activity showed a

270 modulation around the time of water port entry, an excitation when the choice was correct, and

271 an inhibition when the choice was incorrect, even before the mice received a feedback. These

272 “choice commitment"-related signals will be further analyzed below. Finally, delivery of water

273 caused a strong excitation which was modulated by the reward size (Figures $2 \mathrm{C}$ and $2 \mathrm{~F}$ ).

274 Furthermore, the responses to medium-sized water was slightly but significantly smaller on the

275 BIG side compared to the SMALL side (Figures 2C and 2F). The contribution of water-locked

276 kernels was larger than other kernels except in DMS, where odor, movement and water kernels

277 contributed similarly (Figures 2D and 2E).

In previous studies, RPE-related signals have typically been characterized by phasic responses to reward-predictive cues and a delivery or omission of reward. Overall, the above results

281 demonstrate that observed populations contain the basic response characteristics of RPEs. First,

282 dopamine axons were excited by reward-predicting odor cues, and the magnitude of the response

283 was stronger for odors that instructed the animal to go to the side which is associated with a

284 higher value (i.e. BIG side). Responses to water were modulated by reward amounts, and the 285 water responses were suppressed by higher reward expectation. These characteristics were also 286 confirmed by using the actual responses, instead of obtained kernel models (Figures $2 \mathrm{~F}$ and 2G).

287 Finally, in error trials, dopamine axons were inhibited when the time passed beyond the expected 288 time of reward, as the negative outcome becomes certain (Figure 2C). Next, we will investigate 289 each striatal area in more detail. 
293 Although excitation to unpredicted reward is one of the signatures of dopamine RPE, recent

294 studies found that the dopamine axon response to water is small or undetectable in some part of

295 the dorsal striatum (Howe and Dombeck, 2016; Parker et al., 2016; da Silva et al., 2018).

296 Therefore, the above observation that all three areas (VS, DMS, and DLS) exhibited modulation

297 by reward may appear at odds with previous studies.

We noticed greatly diminished water responses when the reward amount was not manipulated, that is, when dopamine axon signals were monitored during training sessions before introducing the reward amount manipulations (Figure 3). In these sessions, dopamine axons in some animals did not show significant excitation to water rewards (Figures 3A and 3D). This "lack" of reward response was found in DMS, consistent with previous studies (Parker et al., 2016), but not in VS or DLS (Figure 3G). Surprisingly, however, DMS dopamine axons in the same animals showed clear excitation when reward amount manipulations were introduced, particularly strongly responding to a big reward (Figures $3 \mathrm{~B}$ and $3 \mathrm{E}$ ). Indeed, the response patterns were qualitatively similar across different striatal areas (Figure 4); the reward responses in all the areas were modulated by reward size and expectation, although the whole responses seem to be shifted higher in DLS, and lower in DMS (Figures 4A and 4B). These results indicate that a stochastic nature of reward delivery in our task enhanced or "rescued" reward responses in dopamine axons

311 in DMS.

313 The above results emphasized the overall similarity of reward responses across areas, but some 314 important differences were also observed. Most notably, although a delivery of a small reward 315 caused an inhibition of dopamine axons below baseline in VS and DMS, the activity remained non-negative in DLS. The overall responses tended to be higher in DLS.

318 In order to understand the diversity of dopamine responses to reward, we examined modulation 319 of dopamine activity by different parameters (Figure 4D). First, the effect of the amount of 320 "actual" reward was quantified by comparing responses to different amounts of water for a given 321 cue (i.e. the same expectation). The reward responses in all areas were modulated by reward amounts, with a slightly higher modulation by water amounts in VS (Figure 4D Water big- 
medium, Water medium-small). Next, the effect of expectation was quantified by comparing the responses to the same amounts of water with prediction of different amounts. Effects of reward size prediction were not significantly different across areas, although VS showed slightly less modulation with more variability (Figure 4D, prediction SMALL-BIG).

Next, we sought to characterize these differences between areas in simpler terms by fitting response curves (response functions). Previous studies that quantified responses of dopamine neurons to varied amounts of reward under different levels of expectation indicated that their reward responses can be approximated by a common function, with different levels of expectation just shifting the resulting curves up and down while preserving the shape (Eshel et al., 2016). We, therefore, fitted dopamine axon responses with a common response function (a power or linear function) for each expectation level (i.e. separately for BIG and SMALL) while fixing the shape of the function (i.e. the exponent of the power function or the slope of the linear function were fixed, respectively) (Figure 4C, Figure S2A). The obtained response functions for the three areas recapitulated the main difference between VS, DMS and DLS, as discussed above. For one, the response curves of DLS are shifted overall upward. This can be characterized by estimating the amount of water that does not elicit a change in dopamine responses from baseline firing ("zero-crossing point" or reversal point). The zero-crossing points, obtained from the fitted curves, were significantly lower in DLS (Figures 4C and 4D). The results were similar regardless of whether the response function was a power (power function $\alpha<1$ ) or a linear

343 function $(\alpha=1)$ (Figure S2B). Similar results were obtained using the aforementioned kernel models in place of the actual activity (Figure S2D).

346 Since the recording locations varied across animals, we next examined the relationship between 347 recording locations and the zero-crossing points (Figures $4 \mathrm{E}$ and $4 \mathrm{~F}$ ). The zero-crossing points

348 varied both along the medial-lateral and the dorsal-ventral axes (linear regression coefficient; $\beta=$ $349-44$ [zero-crossing point water amounts $/ \mathrm{mm}$ ], $\mathrm{p}=0.008$ for medial-lateral axis; $\beta=-52, \mathrm{p}=$ $350 \quad 0.011$ for the dorsal-ventral axis). Examination of each animal confirmed that DMS showed 351 higher zero-crossing points (upper-left in Figure 4E left) whereas DLS showed lower zero352 crossing points (upper-right cluster in Figure 4E right). 
We next examined whether the difference in zero-crossing points manifested specifically during

355 reward responses or whether it might be explained by recording artifacts; upward and downward shifts in the response function can be caused by a difference in baseline activity before trial start

357 (odor onset), and/or lingering activity of pre-reward activity owing to the relatively slow

358 dynamics of the calcium signals (a combination of calcium concentration and the indicator). To

359 examine these possibilities, the same analysis was performed after subtracting the pre-reward

360 signals (Figure S2C). We observed similar or even bigger differences in zero-crossing points

$361\left(\mathrm{p}=2.2 \times 10^{-5}\right.$, analysis of variance [ANOVA] $)$. These results indicate that the elevated or

362 decreased responses, characterized by different zero-crossing points, was not due to a difference

363 in "baseline" but was related to the difference that manifests specifically in responses to reward.

Considerably small zero-crossing points in dopamine axons in DLS were not due to a poor sensitivity to reward amounts nor a poor modulation by expected reward (Figure 4D). Different zero-crossing points, i.e. shifts of the boundary between excitation and inhibition at reward, suggest biased representation of TD error in dopamine axons across the striatum. In TD error models, difference in zero-crossing points may affect not only water responses but also responses to other events. Thus, the small zero-crossing points in dopamine axons in DLS should yield

371 almost no inhibition following an event that is worse than predicted. To test this possibility, we

372 examined responses to events with lower value than predicted (Figure 5): small water (Figures

$3735 \mathrm{~A}-5 \mathrm{C}$ ), water omission caused by choice error (Figures 5D-5F), and a cue that was associated

374 with no outcome (Figures 5G-5I). Consistent with our interpretation of small zero-crossing

375 points, dopamine axons in DLS did not show inhibition in response to outcomes that were worse

376 than predicted while being informative about water amounts.

Taken together, these results demonstrate that dopamine reward responses in all three areas

379 exhibited characteristics of RPEs. However, relative to canonical responses in VS, the responses were shifted more positively in the DLS and more negatively in the DMS. 
The analyses presented so far mainly focused on phasic dopamine responses time-locked to cues and reward. However, dopamine axon activity also exhibited richer dynamics between these events, which need to be explained. For instance, the signals diverged between correct and error trials even before the actual outcome was revealed (a reward delivery versus a lack thereof) (Figure 2C Choice). This difference between correct and error trials, which is dependent on the strength of sensory evidence (or stimulus discriminability), was used to study how neuronal responses are shaped by "confidence". Confidence is defined as the observer's posterior probability that their decision is correct given their subjective evidence and their choice (P(reward|stimulus, choice)) (Hangya et al., 2016). A decision model allows the experimenter to link stimulus discriminability to subjective evidence (Hangya et al., 2016). A given model and task structure makes specific predictions on the shape of three key signatures relating stimulus discriminability, choice and confidence. The predictions can vary depending on task design (Adler and Ma, 2018; Rausch and Zehetleitner, 2019), but the structure of our task follows the original predictions (Hangya et al., 2016). Additionally, in our task, the mice combined the information about reward size with the strength of sensory evidence to select an action (confidence, or uncertainty) (Figure 1). The previous analyses did not address how these different types of information affect dopamine activity over time. We next sought to examine the time course of dopamine axon activity in greater detail, and to determine whether a simple model could explain these dynamics.

Our task design included two delay periods, imposed before choice movement and water delivery, to improve our ability to separate neuronal activity associated with different processes

407 (Figure 1A). The presence of stationary moments before and after the actual choice movement 408 allows us to separate time windows before and after the animal's commitment to a certain option. 409 We examined how the activity of dopamine neurons changed before choice movement and after 410 the choice commitment (Figure 6).

412 We first examined dopamine axon activity after water port entry (0-1 s after water port entry). In 413 this period, the animals have committed to a choice and are waiting for the outcome to be 414 revealed. Responses following different odor cues were plotted separately for trials in which the 415 animal chose the BIG or SMALL side. The vevaiometric curve (a plot of responses against 
416

417

418

419

420

421

422

423

424

425

426

427

428

429

430

431

432

433

434

435

436

437

438

439

440

441

442

443

444

445

446

sensory evidence) followed the expected 'X-pattern' with a modulation by reward size (Hirokawa et al., 2019), which matches the expected value for these trial types, or the size of reward multiplied by the probability of receiving a reward, given the presented stimulus and choice (Figure 6C). The latter has been interpreted as the decision confidence, $P($ reward|stimulus, choice) (Lak et al., 2017, 2020b). The crossing point of the two lines forming an " $\mathrm{X}$ " is shifted to the left in our data because of the difference in the reward size (Figure 6C).

When this analysis was applied to the time period before choice movement $(0-1 \mathrm{~s}$ before odor port exit), the pattern was not as clear; the activity was monotonically modulated by the strength of sensory evidence (\%Odor BIG) only for the BIG choice trials, but not for the SMALL choice trials (Figure 6B). This result is contrary to a previous study that suggested that the dopamine activity reflecting confidence develops even before a choice is made (Lak et al., 2017). We note, however, that the previous study only examined the BIG choice trials, and the results were shown by "folding" the x-axis, that is, by plotting the activity as a function of the stimulus contrast (which would correspond to $\mid \%$ Odor BIG - 50| in our task), with the result matching the so-called "folded X-pattern". We would have gotten the same result, had we plotted our results in the same manner excluding the SMALL choice trials. Our results, however, indicate that a full representation of "confidence" only becomes clear after a choice commitment, leaving open the question what the pre-choice dopamine activity really represents.

The aforementioned analyses, using either the kernel regression or actual activity showed that cue responses were modulated by whether the cue instructed a choice toward the BIG or SMALL side (Figures $2 \mathrm{C}$ and $2 \mathrm{~F}$ ). These results indicate that the information about stimulus-associated values (BIG versus SMALL) affected dopamine neurons earlier than the strength of sensory evidence (or confidence). We next examined the time course of how these two variables affected dopamine axon activity more closely. We computed the dopamine axon activity between trials when a pure odor instructed to go to the BIG versus SMALL side. Consistent with the above result, the difference was evident during the cue period, and then gradually decreased after choice movement (Figure 6D). We performed a similar analysis, contrasting between easy and difficult trials (i.e. the strength of sensory evidence). We computed the difference between 
447 dopamine axon activity in trials when the animal chose the SMALL side after the strongest

448 versus weaker stimulus evidence (a pure odor that instruct to choose the SMALL side versus an

449 odor mixture that instruct to choose the BIG side). In stark contrast to the modulation by the

450 stimulus-associated value (BIG versus SMALL), the modulation by the strength of stimulus

451 evidence in SMALL trials fully developed only after a choice commitment (i.e. water port entry)

452 (Figure 6E). Across striatal regions, the magnitude and the dynamics of modulation due to

453 stimulus-associated values and the strength of sensory evidence were similar (Figures $6 \mathrm{~F}$ and

$4546 \mathrm{G})$, although we noticed that dopamine axons in DMS showed slightly higher correlation with

455 sensory evidence before choice (Figure S3).

457 As discussed above, a neural correlate of "confidence" appears at a specific time point (after

458 choice commitment and before reward delivery) or in a specific trial type (when an animal would

459 choose BIG side) before choice. We, therefore, next examined whether a simple model can

460 account for dopamine axon activity more inclusively (Figure 7). To examine how the value and

461 RPE may change within a trial, we employed a Monte-Carlo approach to simulate animal's

462 choices assuming that the animal has already learned the task. We used a Monte-Carlo method to

463 obtain the ground truth landscape of the state values over different task states, without assuming

464 a specific learning algorithm.

466 The variability and errors in choice in psychophysical performance are thought to originate in the

467 variability in the process of estimating sensory inputs (perceptual noise) or in the process of

468 selecting an action (decision noise). We first considered a simple case where the model contains

469 only perceptual noise (Green and Swets, 1966). In this model, an internal estimate of the

470 stimulus or a "subjective odor" was obtained by adding Gaussian noise to the presented odor

471 stimulus on a trial-by-trial basis (Figures 7B left). In each trial, the subject chooses

472 deterministically the better option (Figure 7C left) based on the subjective odor and the reward

473 amount associated with each choice (Figure 7B right). The model had different "states"

474 considering $\mathrm{N}$ subjective odors ( $\mathrm{N}=60$ and 4 were used and yielded similar results), the

475 available options (left versus right), and a sequence of task events (detection of odor, recognition

476 of odor identity, choice movement, water port entry [choice commitment], Water/No-water

477 feedback, inter-trial interval [ITI]) (Figure 7A). The number of available choices is two after 
478 detecting an odor but reduced to 1 (no choice) after water port entry. In each trial, the model

479 receives one of the four odor mixtures, makes a choice, and obtains feedback (rewarded or not).

480 After simulating trials, the state value for each state was obtained as the weighted sum of

481 expected values of the next states, which was computed by multiplying expected values of the

482 next states with probability of transitioning into the corresponding state. After learning, the state

483 value in each state approximates the expected value of future reward, sum of the amount of

484 reward multiplied by probability of the reward (for simplicity, we assumed no temporal

485 discounting of value within a trial). After obtaining state values for each state, state values for

486 each odor ("objective" odor presented by experimenters) was calculated as the weighted sum of

487 state values over subjective odors. After obtaining state values at each state, we then computed

488 TD errors using a standard definition of TD error which is the difference between the state values

489 at consecutive time points plus received rewards at each time step (Sutton and Barto, 1987).

We first simulated the dynamics of state values and TD errors when the model made a correct choice in easy trials, choosing either the BIG or SMALL side (Figure 7F bottom, blue versus red). As expected, the state values for different subjective odors diverged as soon as an odor identity was recognized, and the differences between values stayed constant as the model received no further additional information before acquisition of water. TD errors, which are the derivative of state values, exhibited a transient increase after odor presentation, and then returned to their baseline levels (near zero), remaining there until the model received a reward. Next, we examined how the strength of sensory evidence affected the dynamics of value and TD errors (Figures 7F and 7J). Notably, after choice commitment, TD error did not exhibit the additional modulation by the strength of sensory evidence, or a correlate of confidence (Figures 7F right and $7 \mathrm{~J}$ right), contrary to our data (Figures $7 \mathrm{E}$ and $7 \mathrm{I}$ right). Thus, this simple model failed to explain aspects of dopamine axon signals that we observed in the data.

504 In the first model, we assumed that the model picks the best option given the available information in every trial (Figure 7C). In this deterministic model, all of the errors in choice are

506 attributed to perceptual noise. We next considered a model that included decision noise in 507 addition to the perceptual noise (Figure 7D). Here decision noise refers to some stochasticity in the action selection process, and may arise from errors in an action selection mechanism or 
exploration of different options, and can be modeled using different methods or rationale behind it. Here we present results based on a "softmax" decision rule, in which a decision variable (in this case, the difference in the ratio of the expected values at the two options) was transformed into the probability of choosing a given option using a sigmoidal function (e.g. Boltzmann

513 distribution) (Sutton and Barto, 2011). We also tested other stochastic decision rules such as

514 Herrnstein's matching law (Herrnstein, 1961) or $\varepsilon$-greedy exploration (randomly selecting an

515 action in a certain fraction $[\varepsilon]$ of trials) (Sutton and Barto, 2011) (Figures S4A-S4C).

Interestingly, just by adding some stochasticity in action selection, various peculiar features of

518 dopamine axon signals described above were suddenly explained (Figures $7 \mathrm{G}$ and $7 \mathrm{~K}$ ). Note that the main free parameters of the above models are the width of the Gaussian noise, which determines the "slope" of the psychometric curve, and was chosen based merely on the

521 behavioral performance, but not the neural data. When the model chose the BIG side, state value at odor presentation was roughly monotonically modulated by the strength of sensory evidence

523 similar to the above (Figure 7G top left). When the model chose the SMALL side, however, the 524 relationship between the strength of sensory evidence and value was more compromised (Figure

$5257 \mathrm{G}$ middle left). As a result, TD error did not show monotonic relationship with sensory

526 evidence before choice (Figures $7 \mathrm{G}$ middle right and $7 \mathrm{~K}$ left), similar to actual dopamine axons

527 responses (Figures 7E middle and 7I left), which was reminiscent of reaction time pattern

528 (Figure 7H). On the other hand, once a choice was committed, the model exhibited interesting

529 dynamics very different from the above deterministic model. After choice commitment, expected

530 value was monotonically modulated by the strength of sensory evidence both for the choice to

531 the BIG and SMALL sides (Figure 7G top and middle left, After). Further, because of the

532 introduced stochasticity in action selection, the model sometimes chose a suboptimal option,

533 resulting in a drop in the state value. This, in turn, caused TD error to exhibit an "inhibitory dip" 534 once the model "lost" a better option (Figure 7G right), similar to the actual data (Figures 7E and 535 7I). This effect was strong particularly when the subjective odor instructed the BIG side but the 536 model ended up choosing the SMALL side. For a similar reason, TD error showed a slight 537 excitation when the model chose a better option (i.e. lost a worse option). The observed features 538 in TD dynamics were not dependent on exact choice strategy: softmax, matching, and $\varepsilon$-greedy, 539 all produced similar results (Figures S4B and S4C). This is because, with any strategy, after 
540 commitment of choice, the model loses another option with a different value, which results in a

541 change in state value. These results are in stark contrast to the first model in which all the choice

542 errors were attributed to perceptual noise.

544 In summary, we found that a standard TD error, computing the moment-by-moment changes in

545 state value (or, the expected future reward), can capture various aspects of dynamics in dopamine

546 axon activity observed in the data, including the changes that occur before and after choice

547 commitment, and the detailed pattern of cue-evoked responses. These results were obtained as

548 long as we introduced some stochasticity in action selection (decision noise), regardless of how

549 we did it. The state value dynamically changes during the performance of the task because the

550 expected value changes according to an odor cue (i.e. strength of sensory evidence and stimulus-

551 associated values) and the changes in potential choice options. A drop of the state value and TD

552 error at the time of choice commitment occurs merely because the state value drops when the

553 model chose an option that was more likely to be an error. Further, a correlate of "confidence"

554 appears after committing a choice, merely because at that point (and only at that point), the state

555 value becomes equivalent to the reward size multiplied with the confidence, i.e. the probability

556 of reward given the stimulus and the choice. This means that, as long as the animal has

557 appropriate representations of states, a representation of "confidence" can be acquired through a

558 simple associative process or model-free reinforcement learning without assuming other

559 cognitive abilities such as belief states or self-monitoring (meta-cognition). In total, not only the

560 phasic responses but also some of the previously unexplained dynamic changes can be explained

561 by TD errors computed over the state value, provided that the model contains some stochasticity

562 in action selection in addition to perceptual noise. Similar dynamics across striatal areas (Figure

563 6) further support the idea that dopamine axon activity follows TD error of state values in spite

564 of the aforementioned diversity in dopamine signals. 


\section{DISCUSSION}

568 In the present study, we monitored dopamine axon activity in three regions of the striatum (VS, 569 DMS and DLS) while mice performed instrumental behaviors involving perceptual and value570 based decisions. In addition to phasic responses associated with reward-predictive cues and

571 reward, we also analyzed more detailed temporal dynamics of the activity within a trial. Contrary

572 to the current emphases on diversity or multiplexing in dopamine signals (and therefore, to our

573 surprise), we found that dopamine axon activity in all of the three areas exhibited dynamics that

574 can be explained by the TD error which calculates moment-by-moment "changes" in the

575 expected future reward (i.e. state value). Interestingly, however, our results showed consistent 576 differences between regions. First, as reported previously (Parker et al., 2016), during choice 577 movements, contra-lateral orienting movements caused a transient activation in the DMS. This

578 response was negligible in VS and DLS, however. Second, although dopamine axon signals 579 exhibited temporal dynamics that are predicted by TD errors, reward responses were generally

580 elevated in DLS. As a consequence, dopamine axon signals in DLS did not exhibit a clear 581 inhibitory response (“dopamine dip") even when the actual reward was smaller than expected, or 582 even when the animal did not receive a reward, despite our observations that dopamine axons in 583 VS and DMS exhibited clear inhibitory responses in these conditions. Overall, the activity during 584 the reward period was biased toward positive responses in the DLS, compared to other areas.

585 Activation of dopamine neurons both in VTA and SNc are known to reinforce preceding 586 behaviors (Ilango et al., 2014; Keiflin et al., 2019; Lee et al., 2020; Saunders et al., 2018). The 587 differences in dopamine axon signals that we observed in instrumental behaviors can provide 588 specific constraints on the behaviors learned through dopamine-mediated reinforcement in these 589 striatal regions.

\section{Diversity in representation of TD errors}

593 Accumulating evidence indicates that dopamine neurons are diverse in various aspects such as 594 anatomy, physiological properties, and activity (Engelhard et al., 2019; Farassat et al., 2019; 595 Howe and Dombeck, 2016; Kim et al., 2015; Lammel et al., 2008; Matsumoto and Hikosaka, 596 2009; Menegas et al., 2015, 2017, 2018; Parker et al., 2016; da Silva et al., 2018; Watabe-Uchida 
597

598

599

600

601

602

603

604

605

606

607

608

609

610

611

612

613

614

615

616

617

618

619

620

621

622 projection-specific manner.

623

624 Higher criteria in DMS may partly explain the observation that some dopamine neurons do not 625 show a clear excitation by reward, such as in the case of our recording without reward amount

626 modulations (Figure 3). Our results suggest that whether dopamine neurons respond to reward 627 likely depends critically on task structures and training history. It will be important to further 
628

629

630

631

632

633

634

635

636

637

638

639

640

641

642

643

644

645

646

647

648

649

650

651

652

653

654

655

656

657

658

examine in what conditions these dopamine neurons lose responses to water, or whether there are dopamine neurons which do not respond to reward in any circumstances. In contrast to DMS, we observed reliable excitation to water reward in dopamine axons in DLS. Thus, the previous observation that some dopamine neurons in the substantia nigra show small or no excitation to reward (da Silva et al., 2018) may mainly come from DMS-projecting dopamine neurons or another subpopulation of dopamine neurons that project to the tail of the striatum (TS) (Menegas et al., 2018), but not DLS. The distinction is important because smaller dopamine responses to reward have been often linked to skill or habit with value-free mechanism (Miller et al., 2019). In contrast, we found that dopamine axons in DLS show strong modulation by reward amounts and prediction, and its dynamics resemble TD errors. Our observation suggests that the lack of reward omission responses and excitation by even small rewards is a key for the function of dopamine in DLS.

\section{Positively biased reinforcement signals in DLS dopamine}

It has long been observed that the activity of many dopamine neurons exhibits a phasic inhibition when an expected reward was omitted or when the reward received was smaller than expected (Hart et al., 2014; Schultz et al., 1997). This inhibitory response to negative RPEs is one of the hallmarks of dopamine RPE signals. Our results that DLS dopamine signals largely lack these dopamine dips (Figure 4 and Figure 5) has profound implications on what types of behaviors are learned through DLS dopamine signals as well as what computational principles underlie reinforcement learning in DLS.

Dopamine "dips" are thought to act as aversive stimuli and/or can facilitate extinction of previously learned behaviors (weakening) (Chang et al., 2018; Montague et al., 1996; Schultz et al., 1997). The lack of dopamine dip in DLS may lead to the animal's reduced sensitivity to worse-than-expected outcome (i.e. negative prediction error). This characteristic resembles the activity of dopamine axons in TS, posterior to DLS, which signals potential threat and also lacks inhibitory responses to an omission of a predicted threat (Menegas et al., 2017, 2018). We proposed that the lack of inhibitory omission signals (and so lack of weakening signals) would 
659

660

661

662

663

664

665

666

667

668

669

670

671

672

673

674

675

676

677

678

679

680

681

682

683

684

685

686

687

688

689

be critical to maintain threat prediction even if an actual threat is sometimes omitted. Similarly, the lack of weakening signals in DLS may help keep the learned actions from being erased even if the outcome is sometimes worse than predicted or even omitted. This idea is in line with the previous observations that DLS plays an important role in habitual behaviors (Yin et al., 2004). The uniquely modified TD error signal in DLS (i.e. a reduced inhibitory response during the reward period) may explain a predominant role of DLS in controlling habitual behaviors.

\section{What is learned in the DLS? "The law of exercise" and learning sequences.}

A deeper understanding of the nature of reinforcement signals can constrain the search for computational principles and provide critical insight into what is actually learned by the system.

Here we speculate on these questions in the light of reinforcement learning theories and anatomy.

Thorndike (Thorndike, 1932) proposed three principles for instrumental learning - the law of effect, the law of readiness, and the law of exercise. The law of effect emphasizes the role of outcome of behaviors: behaviors that led to good outcomes become more likely to occur - the idea that is a foundation of value-based reinforcement learning. In contrast, the law of exercise emphasizes the number of times a particular action was taken. There has been an increasing appreciation of the law of exercise because repetition or overtraining is the hallmark of habits and skills (Hikosaka et al., 1995; Matsuzaka et al., 2007; Miller et al., 2019; Morris and Cushman, 2019; Ölveczky, 2011; Robbins and Costa, 2017; Smith and Graybiel, 2016). Here we propose that dopamine signals in DLS provide an ideal neural substrate of learning with an emphasis on the law of exercise. A positively biased TD error signals ensures that an "OK" action will be positively reinforced, in a manner that depends on the number of times that the same behavior was repeated as far as it is accompanied by a small reward (i.e. with "OK" signals). This property may explain why the formation of habit (and skills) normally requires overtraining (i.e. repeating a certain behavior many times).

The observation that DLS dopamine signals lack inhibitory responses raises the question what is actually learned by the system. Learning of values depends on the balance between positive and 
690

691

692

693

694

695

696

697

698

699

700

701

702

703

704

705

706

707

708

709

710

711

712

713

714

715

716

717

718

719

720

negative prediction errors: the learned value converges to the point at which positive and negative prediction errors form an equilibrium. If a reinforcement signal lacks negative prediction errors, this learning would no longer work as it was originally conceptualized. In reinforcement learning theories, an alternative approach is policy-based reinforcement learning. We propose that policy learning may be a better way to conceptualize the function of the DLS. In reinforcement learning, a policy is a set of rules that map an action to a state, and has direct relevance to stimulus-response associations that are proposed to underlie habit because the relationship between stimulus (state) and response (action) can be more directly encoded using a policy. According to Sutton and Barto (Sutton and Barto, 2018), policy learning can be done by learning what is called "preference", $h(s, a)$, which defines the likelihood of a certain action, $a$, in a given state, $s$. In a given state, an action is selected based on preference through a winnertake-all mechanism either deterministically (e.g. by selecting the action with the maximum preference) or stochastically (e.g. through a softmax action selection). One way to conceptualize preference is to see it as a generalized version of value, which has less constraints than value (the idea of "value" may imply many properties that it should follow, e.g. the value should be zero for no outcome). Alternatively, $h(s, a)$ can directly encode the probability of an action.

It is also important to consider what are "states" for learning in DLS. Importantly, the main inputs to DLS come from the motor cortex, somatosensory cortex, and other subcortical areas such as intralaminar nuclei in thalamus (Hunnicutt et al., 2016). Thus, the inputs to DLS may not be dominated by the sensory information representing the external world, as often conceptualized in reinforcement learning. Instead, DLS is well-positioned to receiving inputs representing motor commands (the current "motor states") or somatosensory information (the current "bodily states" consisting of proprioception, sense of touch etc.). In other words, DLS may compute their output by monitoring the current motor and bodily states. Dopamine in DLS can thus be conceptualized as a reinforcement signal that strengthens the connection between the current motor/bodily state and the next motor output. This mechanism, when chained, can produce a sequence of movements as long as the same motor/bodily state is revisited or reproduced, which may not occur easily at the beginning but can occur after repeated training. As such, DLS may regulate "how" to perform a sequence of well-trained movements smoothly and automatically. The key properties of habits and skills such as stereotypy, automaticity and 
721 the requirement of overtraining, can be explained by this model. In this model, the learning of

722 habits and skills are a natural consequence of reinforcement learning using a specialized

723 reinforcement signals (positively shifted response to outcomes) and the unique anatomical

724 property (the specialized input suitable for chaining actions) of the DLS. Future experiments

725 using tasks involving sequence of actions (Hikosaka et al., 1995; Ölveczky, 2011) can test this

726 idea.

\section{Potential mechanisms underlying diverse TD error signals}

We found that, across the striatum, dopamine signals overall resemble TD errors, with positive or negative biases in a subregion-specific manner (Figure 4). A potential mechanism to generate such a diversity is by optimistic and pessimistic expectations, as proposed in distributional reinforcement learning (Dabney et al., 2020). Alternatively, DLS-projecting dopamine neurons may add "success premium" at each feedback. Signals of success feedback were observed in multiple cortical areas (Chen et al., 2017; Sajad et al., 2019; Stuphorn et al., 2000), which is often more sustained than phasic dopamine responses. Interestingly, we noticed that responses to

737 water in dopamine axons in DLS are more sustained than dopamine axons in other areas (Figure

738 4A). DLS-projecting dopamine neurons potentially receive and integrate those success feedback 739 signals with reward value, shifting the teaching signals more positively.

Mechanistically, biases in dopamine signals may stem from a difference in the excitation-

742 inhibition balance at the circuit level. In addition to dopamine neurons, there are multiple brain

743 areas where activity of some neurons resembles RPE (Li et al., 2019; Matsumoto and Hikosaka,

744 2007; Oyama et al., 2010; Tian et al., 2016). Among these, presynaptic neurons in multiple brain 745 areas directly convey a partial prediction error to dopamine neurons (Tian et al., 2016). On the 746 other hand, rostromedial tegmental area (RMTg) exhibits a flipped version of RPE (the sign is

747 opposite to dopamine neurons), and its inhibitory neurons directly project to dopamine neurons 748 in a topographic manner (Hong et al., 2011; Jhou et al., 2009a, 2009b; Li et al., 2019; Tian et al., 749 2016). Hence, each dopamine neuron may receive a different ratio of excitatory and inhibitory 750 inputs of RPE. It would be interesting if DLS-projecting dopamine neurons receive less 751 inhibitory RPE, and DMS-projecting dopamine neurons receive more, so that RPE signals are 
752 pushed up or down, whereas the information is still almost intact. In addition to anatomical

753 reasons, DLS-projecting dopamine neurons show higher burstiness in intact animals (Farassat et

754 al., 2019) and higher excitability in vitro (Evans et al., 2017; Lerner et al., 2015). These multiple

755 reasons may explain why DLS-projecting dopamine neurons do not show inhibitory responses to

756 negative prediction errors. It will be fascinating if we could connect all these levels of studies

757 into functional meaning in the future.

758

Future directions to understand the meaning of diversity of dopamine signals

760

Recent studies reported that dopamine neurons are modulated by various parameters (Engelhard et al., 2019; Watabe-Uchida and Uchida, 2018). Here, we found that TD error dynamics can inclusively explain two seemingly separate decision variables, namely, stimulus-associated value and choice accuracy when animal's choice strategy is not deterministic (i.e. there is decision noise) (Figure 6). At a glance, dopamine activity patterns may appear to be signaling two distinct variables at different timings, but both are inclusively explained by a single quantity (TD error)

767 in one framework (Figure 7). These results underscore the importance of considering moment-

768 by-moment dynamics, and underlying computation. Taken together, our results showed that 769 dopamine axon signals in the striatum approximate TD error dynamics. We propose that 770 dopamine in different striatal areas conveys TD errors in a biased manner. One compelling idea

771 is that the lack of negative teaching signals in DLS plays a role in skill/habit, although further

772 examination is needed to establish its functions. Although we designed the task to minimize

773 effects of movement itself on results, accumulating studies suggested close relationship between

774 dopamine signaling and movement (Howe and Dombeck, 2016; da Silva et al., 2018). It is

775 important to test these other parameters in the future in order to understand the meaning of the

776 diversity of dopamine neurons and organization of dopamine-striatum systems. 


\section{EXPERIMENTAL PROCEDURES}

\section{Animals}

785

17 dopamine transporter (DAT)-cre (B6.SJL-Slc6a3tm1.1(cre)Bkmn/J, Jackson Laboratory; RRID:IMSR JAX:006660) (Bäckman et al., 2006) heterozygous mice, and 5 DAT-Cre;Ai14 (Rosa-CAG-LSL-tdTomato, Jackson Laboratory; RRID:IMSR JAX:007914) (Madisen et al., 2010) double heterozygous mice, male and female, were used for recording signals from dopamine axons. All mice were backcrossed with C57BL/6J (Jackson Laboratory). Animals were housed on a 12 hour dark/12 hour light cycle (dark from 07:00 to 19:00) and performed a task at the same time each day. All procedures were performed in accordance with the National Institutes of Health Guide for the Care and Use of Laboratory Animals and approved by the Harvard Animal Care and Use Committee.

\section{Surgical Procedures}

All surgeries were performed under aseptic conditions with animals anesthetized with isoflurane (1-2\% at 0.5-1.0 1/min). Analgesia was administered pre (buprenorphine, $0.1 \mathrm{mg} / \mathrm{kg}$, I.P) and postoperatively (ketoprofen, $5 \mathrm{mg} / \mathrm{kg}$, I.P). To express GCaMP7f (Dana et al., 2019) specifically in dopamine neurons, we unilaterally injected $300 \mathrm{nl}$ of mixed virus solution; AAV5-CAGFLEX-GCaMP7f $\left(1 \times 10^{12}\right.$ particles/ml, UNC Vector Core, NC) and AAV5-CAG-FLEXtdTomato $\left(2 \times 10^{13}\right.$ particles/ml, UNC Vector Core, NC) into both the VTA and SNc (600 nl total) in the DAT-cre mice. Only AAV5-CAG-FLEX-GCaMP7f (300 nl total) was used for DAT;Ai14 double transgenic mice. Virus injection lasted around 20 minutes, and then the injection pipette was slowly removed over the course of several minutes to prevent damage to the tissue. We also implanted optic fibers (400 $\mu \mathrm{m}$ diameter, Doric Lenses, Canada) into the VS,

806 DMS, or DLS (1 fiber per mouse). To do this, we first slowly lowered optical fibers into the 807 striatum. Once fibers were lowered, we first attached them to the skull with UV-curing epoxy 808 (NOA81, Thorlabs, NJ), and then a layer of rapid-curing epoxy to attach the fiber cannulas even 809 more firmly to the underlying glue. After waiting 15 minutes for this to dry, we applied a black 810 dental adhesive (Ortho-Jet, Lang Dental, IL). We used magnetic fiber cannulas (Doric Lesnses, 811 MFC_400/430) and the corresponding patch cords to allow for recordings in freely moving 
812 animals. After waiting 15 minutes for the dental adhesive to dry, the surgery was complete. We

813 used the following coordinates to target our injections and implants.

814

815 - $\quad$ (VTA) Bregma: $-3.0 \mathrm{~mm}$, Lateral: $0.6 \mathrm{~mm}$, Depth: between $4.5 \mathrm{~mm}$ and $4.3 \mathrm{~mm}$

816 - $\quad$ (SNc) Bregma: -3.0 mm, Lateral: $1.6 \mathrm{~mm}$, Depth: between $4.3 \mathrm{~mm}$ and $4.1 \mathrm{~mm}$

817 - (VS) Bregma: between $1.5 \mathrm{~mm}$ and $1.0 \mathrm{~mm}$, Lateral: $1.8 \mathrm{~mm}$, Depth: $3.8 \mathrm{~mm}$, angle 10

818 - (DMS) Bregma: between $1.5 \mathrm{~mm}$ and $0 \mathrm{~mm}$, Lateral: $1.3 \mathrm{~mm}$, Depth: $2.3 \mathrm{~mm}$

819 - (DLS) Bregma: between $1.3 \mathrm{~mm}$ and -0.8 mm, Lateral: $3.0 \mathrm{~mm}$, Depth: $2.3 \mathrm{~mm}$

\section{Behavioral tasks}

822 All behavioral experiments were performed in custom-built behavioral rigs and controlled by a

823 NIDAQ board (National Instruments, TX) and Labview (National Instruments, TX), similar to a

824 previous study (Uchida and Mainen, 2003). Mice were trained to perform an odor-discrimination

825 task for water reward, similar to a study in rats (Uchida and Mainen, 2003) with several

826 modification. Mice initiated trials in a self-paced manner by poking a center port, which then

827 delivered an odor. Different odors were used in a pseudorandomized order from 3 different pure

828 chemicals (odor A, B and C) and mixtures of odor A and B with various ratios. Mice were

829 required to choose a left or right water port depending on dominant odor identity, odor A or B.

830 Correct choice was always rewarded by a drop of water. Odor C was never associated with

831 outcomes. To isolate cue- and water-related signals from potential motion artifacts in recording

832 and motor-related activity, mice were required to stay in an odor port for at least $1 \mathrm{~s}$, and then to

833 stay in a water port for $1 \mathrm{~s}$ to get water reward. The inter-trial-interval was fixed at $7 \mathrm{~s}$ after water

834 onset in correct trials and at $9 \mathrm{~s}$ after any types of an error including violation of the stay

835 requirement, no choice within $5 \mathrm{~s}$ after odor port out, and multiple pokes of an odor port after

836 odor delivery. 1-Butanol, eugenol and cymene were diluted in 1/10 with mineral oil and

837 randomly assigned to odor A, B or C across animals. The odor-port assignment (left or right) was

838 held constant in a single animal.

840 Mice were first trained only with pure odors and with the same amounts of water reward ( $\sim 6 \mathrm{ul})$.

841 After mice achieved greater than 90\% accuracy, mice received a surgery for viral injection and

842 fiber implantation. Following a 1-week recovery period, mice received re-training and then, 
843 mixtures of odor A and B (100/0, 90/10, 65/35, 35/65, 10/90, 0/100) were gradually introduced.

844 After the accuracy of all the mixture odors achieved more than 50\%, neuronal recording with

845 fiber fluorometry was performed for 5 sessions. Subsequently, a task with different amounts of

846 water was introduced. Mixtures of odor A and B (100/0, 65/35, 35/65, 0/100) but no odor C were

847 used in this task. Each recording session started with 88-120 trials with an equal amount of water

848 ( $\sim 6 \mathrm{ul}$, the standard amount) in the first block to calibrate any potential bias on the day. In the

849 second block, different amounts of reward were delivered in each water port. In order to make

850 the water amounts unpredictable, one water port delivered big or medium size of water (2.2 and

8510.8 times of the standard, $\sim 13.2$ and $4.8 \mu \mathrm{l}$, BIG side) in a pseudo-random order, and another

852 water port delivered medium or small size of water ( 0.8 and 0.2 times of the standard, $\sim 4.8$ and

$8531.2 \mu \mathrm{l}$, SMALL side) in a pseudo-random order. Block 2 continued for 200 trials or until the end

854 of recording sessions, whichever came earlier. A mouse performed 134.3 $\pm 3.4($ mean $\pm \mathrm{SEM})$

855 trials in block 2. The water condition (BIG or SMALL) was assigned to a left or right water port

856 in a pseudo-random order across sessions. Recording was conducted for 40 min every other day

857 to avoid potential bleaching. On days with no recording, animals were trained with pure odors A

858 and B with the standard amount of water.

Fiber photometry

861 Fiber fluorometry (photometry) was performed as previously reported (Menegas et al., 2018)

862 with a few modification. The optic fiber (400 $\mu \mathrm{m}$ diameter, Doric Lenses) allows chronic, stable,

863 minimally disruptive access to deep brain regions and interfaces with a flexible patch cord (Doric

864 Lenses, Canada) on the skull surface to simultaneously deliver excitation light (473 nm,

865 Laserglow Technologies, Canada; 561 nm, Opto Engine LLC, UT) and collect GCaMP and

866 tdTomato fluorescence emissions. Activity-dependent fluorescence emitted by cells in the

867 vicinity of the implanted fiber's tip was spectrally separated from the excitation light using a

868 dichroic, passed through a single band filter, and focused onto a photodetector connected to a

869 current preamplifier (SR570, Stanford Research Systems, CA). During recording, optic fibers

870 were connected to a magnetic patch cable (Doric Lesnses, MFP_400/430) which delivered

871 excitation light (473 $\mathrm{nm}$ and $561 \mathrm{~nm}$ ) and collected all emitted light. The emitted light was

872 subsequently filtered using a 493/574 nm beam-splitter (Semrock, NY) followed by a $500 \pm 20$

$873 \mathrm{~nm}$ (Chroma, VT) and $661 \pm 20 \mathrm{~nm}$ (Semrock, NY) bandpass filters and collected by a 
874 photodetector (FDS10x10 silicone photodiode, Thorlabs, NJ) connected to a current preamplifier

875 (SR570, Stanford Research Systems, CA). This preamplifier output a voltage signal which was

876 collected by a NIDAQ board (National Instruments, TX) and Labview software (National

877 Instruments, TX).

878

879

\section{Histology}

880

Mice were perfused using 4\% paraformaldehyde and then brains were sliced into $100 \mu \mathrm{m}$ thick coronal sections using a vibratome and stored in PBS. Slices were then mounted in anti-fade solution (VECTASHIELD anti-fade mounting medium, H-1000, Vector Laboratories, CA) and imaged using a Zeiss Axio Scan Z1 slide scanner fluorescence microscope (Zeiss, Germany).

\section{Behavior analysis}

We fitted $\%$ of odor mixture $(\mathrm{X})$ to $\%$ of choice left or choice BIG $(\mu)$ using generalized linear model with logit link function in each animal as previously reported (Uchida and Mainen, 2003). $\log (\mu /(1-\mu))=\mathrm{Xb}_{1}+\mathrm{b}_{0}$

We first fitted a control block (block 1) and a reward-manipulation block (block 2) separately to examine difference of a slope, $b_{1}$ and a bias, $50-b_{0} / b_{1}$ of the curve. Next, to quantify shift of choice bias, we fitted choice of block 1 and block 2 together with a fixed slope, by fitting odor

$892\left(\mathrm{X}_{1}\right)$ and a block type $\left(\mathrm{X}_{2}=0\right.$ for block $1, \mathrm{X}_{2}=1$ for block 2$)$ to choice.

$\log (\mu /(1-\mu))=X_{1} b_{1}+X_{2} b_{2}+b_{0}$

894 Choice bias in block 2 was quantified choice bias as a lateral shift of the psychometric curve 895 equivalent to \% mixture of odors, $50-\left(b_{0}+b_{2}\right) / b_{1}$, which is a lateral shift compared to no bias, and $b_{0} / b_{1}-\left(b_{0}+b_{2}\right) / b_{1}$, which is a lateral shift compared to choice in block 1 .

\section{GCaMP detection and analysis}

899 To synchronize behavioral events and fluorometry signals, TTL signals were sent every $10 \mathrm{~s}$

900 from a computer that was used to control and record task events using Labview, to a NIDAQ

901 board that collects fluorometry voltage signals. GCaMP and tdTom signals were collected as

902 voltage measurements from current preamplifiers. Green and red signals were cleaned by

903 removing $60 \mathrm{~Hz}$ noise with bandstop FIR filter $58-62 \mathrm{~Hz}$ and smoothing with moving average of

904 signals in 50ms. The global change within a session was normalized using a moving median of 
905

906

907

908

909

910

911

912

913

914

915

916

917

918

919

920

921

922

923

924

925

926

927

928

929

930

931

932

933

934

935

100s. Then, the correlation between green and red signals during ITI was examined by linear regression. If the correlation is significant $(\mathrm{p}<0.05)$, fitted tdTom signals were subtracted from green signals.

Responses were calculated by subtracting the average baseline activity from the average activity of the target window. Unless specified otherwise, odor responses were calculated by averaging activity from 1-0 s before odor port out (before choice) minus the average activity from the baseline period (1-0.2 s before odor onset). Responses after choice were calculated by averaging activity from 0-1 s after water port in minus the same baseline. Outcome responses were calculated by averaging activity from $0-1 \mathrm{~s}$ after water onset minus the same baseline. When comparing activity before and after water onset, average activity in 1-0.2 s before water onset was used as baseline. To normalize GCaMP signals across sessions within an animal, GCaMP signals were divided by average of peak responses during $1 \mathrm{~s}$ after odor onset in all the successful trials in the session. Z-scores of the signals were obtained using mean and standard deviation of signals in all the choice trials (from $2 \mathrm{~s}$ before odor onset to $6 \mathrm{~s}$ after odor onset) in each animal.

We built a regularized linear regression to fit cosine kernels (Park et al., 2014) (width of $200 \mathrm{ms,}$ interval of $40 \mathrm{~ms}$ ) to the activity of dopamine axons in each animal. We used down-sampled (every $20 \mathrm{~ms}$ ) responses in all valid choice trials (trials with $>1$ s odor sampling time and any choice, -1 to $7 \mathrm{~s}$ from odor onset) for the model fitting. We used 4 different time points to lock kernels: odor onset ("odor"), odor port out ("movement"), water port in ("choice"), and water onset ("water"). Odor kernels consist of 4 types of kernels: "base" kernels to span -960 to $200 \mathrm{~ms}$ from odor onset in all trials, and "pure big" kernels in trials with a pure odor associated with big/medium water, "pure small" kernels in trials with a pure odor associated with medium/small water, and "mixture" kernels in trials with a mixture odor to span 0-1600 ms from odor onset. Movement kernels consist of 2 types of kernels: "contra turn" kernels in trials with choice contralateral to the recording site, and "ipsi turn" kernels in trials with choice ipsi-lateral to the recording site to span -1000 to $1200 \mathrm{~ms}$ from when a mouse exited an odor port. Choice kernels consist of 3 types of kernels: "correct big" kernels in trials with correct choice of medium/small water and "correct small" kernels in trials with correct choice of medium/small water to span - 
936400 to $1200 \mathrm{~ms}$ from when a mouse entered a water port (water port in), and "error" kernels in

937 trials with choice error to span -400 to $5200 \mathrm{~ms}$ from water port in. Water kernels consist of 4

938 types of kernels: "big water" kernels for big size of water, "medium water big side" kernels for

939 medium size of water at a water port of big/medium water, "medium water small side" kernels

940 for medium size of water at a water port of medium/small water, and "small water" for small size

941 of water to span 0-4200 ms after water onset. All the kernels were fitted to responses using linear

942 regression with Lasso regularization with 10-fold cross validation. Regularization coefficient

943 lambda was chosen so that cross-validation error is minimum plus one standard deviation. \%

944 explained by a model was expressed as reduction of a variance in the residual responses

945 compared to the original responses. Contribution of each component in the model was measured

946 by reduction of a deviance compared to a reduced model excluding the component.

948 We estimated response function to water in dopamine axons with linear regression with power

949 function in each animal.

$$
r=k\left(R^{\alpha}+c 1 * S+c 2\right)
$$

951 where $\mathrm{r}$ is the dopamine axon response to water, $\mathrm{R}$ is the water amount, $\mathrm{S}$ is SMALL side $(\mathrm{S}=1$

952 when water was delivered at SMALL side, $\mathrm{S}=0$ otherwise). There are 4 different conditions,

953 responses to big and medium water at a port of BIG side, and to medium and small water at a

954 port of SMALL side. We first optimized $\alpha$ by minimizing average of residual sum of squares for

955 each animal and then applied $\alpha=0.7$ for all the animals to obtain other parameters, $\mathrm{k}, \mathrm{c} 1$, and c2.

956 The response function was drawn with $\mathrm{R}$ as $\mathrm{x}$-axis and $\mathrm{r}$ as $\mathrm{y}$-axis. The amount of water to which

957 dopamine axons do not respond under expectation of BIG or SMALL water was estimated by

958 getting a crossing point of the obtained response function where the value is 0 (a zero-crossing

959 point). The distribution of zero-crossing points was examined by linear regression of zero-

960 crossing values against anatomical locations (anterior-posterior, dorsal-ventral, and medial-

961 lateral). To visualize zero crossing points on the atlas, zero-crossing values were fitted against

962 anatomical locations with interaction terms using linear regression with elastic net regularization

$963(\alpha=0.1)$ with 3 -fold cross validation. The constructed map was sliced at a coronal plane Bregma

$964+0.7$ and overlaid on an atlas (Paxinos and Franklin, 2019). 
966 To visualize activity pattern in multiple time windows at the same time, we stretched activity in

967 each trial to standard windows. Standard windows from odor onset to odor poke out, and from

968 odor poke out to water poke in, were determined by median reaction time and median movement

969 time for each animal. For average plots of multiple animals, windows were determined by the

970 average of median reaction times and of median movement times in all animals. The number of

971 100ms bins in each time window was determined by dividing median reaction time and median

972 movement time by 100. Dopamine responses in the window were divided into the bin number

973 and the average response in each bin was stretched to 100ms. The stretched activity patterns

974 were used only for visualization, and all the statistical analyses were performed using original

975 responses.

977 Estimation of state values and TD errors using simulations

978 To examine how the value and RPE may change within a trial, we employed a Monte-Carlo

979 approach to simulate animal's choices at a steady state (i.e. after the animal learned the task). We

980 used a Monte-Caro approach to obtain the ground truth state values as the animal progresses

981 through task events without assuming a specific learning algorithm, under the assumption that

982 the animal has learned the task. After obtaining the state values, we computed TD errors over the

983 obtained state values.

Model architecture

986 We considered two types of models. The variability and errors in choice in psychophysical

987 performance can arise from at least two noise sources; noise in the variability in the process of

988 estimating sensory inputs (perceptual noise) and noise in the process of selecting an action

989 (decision noise). The first model contained only perceptual noise (Green and Swets, 1966), and

990 the second model contained both perceptual and decision noise.

992 These models had different "states" considering $\mathrm{N}_{\mathrm{S}}$ subjective odors $\left(\mathrm{N}_{\mathrm{S}}=60\right.$ or 4 discrete

993 states), choice (BIG versus SMALL), and different timing (inter-trial interval, odor port entry,

994 odor presentation, choice, water port in, waiting for reward, and receiving feedback/outcome)

995 (circles in Figure 7A). 
We assumed Ns possible subjective odor states (O’) which comprise SubOdor1 and SubOdor2

998 states. We assumed that, in each trial, an internal estimate of the stimulus or a "subjective odor"

999 (O') was obtained by adding a noise to the presented odor stimulus (O) (one of the 4 mixtures of

1000 Odor A and B; 100/0, 65/35, 35/65, 0/100) (Figure 7A-C). In the model, the probability of falling 1001 on a given subjective odor state (O') is calculated using a Gaussian distribution centering on the 1002 presented odor $(\mathrm{O})$ with the standard deviation, $\sigma$. We considered two successive states for 1003 subjective odor states in order to reflect a relatively long duration before an odor port exit.

As in the behavioral paradigm, whether the model receives a reward or not was determined solely by whether the presented odor (O) instructed the BIG side or SMALL side. Each subjective odor state contains cases when the presented odor $(\mathrm{O})$ is consistent or congruent with the subjective odor $\left(\mathrm{O}^{\prime}\right)$. For each subjective odor state, the probability of receiving a reward after choosing the BIG side, $p(B I G$ is correct $)=f_{B}$, can be calculated as the fraction of cases when the presented odors instructed the BIG side. Conversely, the probability of reward after choosing the SMALL side is $p(S M A L L$ is correct $)=f_{S}=1-f_{B}$. Note that neither $f_{B}$ nor $f_{S}$

1012 depends on reward size manipulations (as will be discussed later, the animal's choices will be 1013 dependent on reward size manipulations).

\section{Action selection}

1016 For each subjective odor, the model chose either the BIG or the SMALL side based on the value 1017 of choosing the BIG or SMALL side $\left(V_{B}\right.$ and $V_{S}$ respectively, equivalent to the state value of the 1018 next state after committing to choose the BIG or SMALL side; see below for how $V_{B}$ and $V_{S}$ 1019 were obtained). In the first model which contains only perceptual noise, the side that is associated with a larger value is chosen. In the second model which contains both perceptual and

1021 decision noise, a choice is made by transforming $V_{B}$ and $V_{S}$ into the probability of choosing a 1022 given option using a sigmoidal function (e.g. Boltzmann distribution) (Sutton and Barto, 2011).

1023 In the softmax, the probabilities of choosing the BIG and SMALL side $\left(P_{B}, P_{S}\right)$ are given,

1024 respectively, by,

$$
\begin{gathered}
P_{B}=\frac{e^{\left(V_{B} /\left(V_{B}+V_{S}\right)\right) / \tau}}{e^{\left(V_{B} /\left(V_{B}+V_{S}\right)\right) / \tau}+e^{\left(V_{S} /\left(V_{B}+V_{S}\right)\right) / \tau}} \\
P_{S}=1-P_{B}
\end{gathered}
$$


1027 We also tested other stochastic decision rules such as Herrnstein's matching law (Herrnstein, 1028 1961) or $\varepsilon$-greedy exploration (randomly selecting an action in a certain fraction [E] of trials) 1029 (Sutton and Barto, 2011). In Herrnstein's matching law, the probability of choosing the BIG side 1030 is given by,

$$
P_{B}=\frac{V_{S}}{V_{S}+V_{B}}
$$

1032

1033 The perceptual noise and a set of decision rule determine the behavioral performance of the 1034 model. The first model has only one free parameter, $\sigma$. The second model has one or no 1035

1036 additional parameter ( $\tau$ for softmax, or $\varepsilon$, for $\varepsilon$-greedy; no additional parameter for matching).

1037 (the average performance in Block 2; i.e. Figure 1C, orange) by minimizing the mean squared 1038 errors in the psychometric curves.

1039

1040 For the first model, the best fit $\sigma$ was $21 \%$ Odor. We also tested with $\sigma$ of $5 \%$, and the TD error 1041 dynamic was qualitatively similar. For the second model using the softmax rule, the best fit $\tau$ 1042 was 0.22 while $\sigma$ was $18 \%$ Odor.

State values

1045 The state value for each state was obtained as the weighted sum of expected values of available 1046 options which was computed by multiplying expected values of the option with probability of an option in the next step.

Outcome 2 state represents the timing when the animal recognizes the amount of water. The state value is given by the amount of water that the model received (big, medium, small),

$$
\begin{aligned}
& V_{b}=2.2^{\alpha} \\
& V_{m}=0.8^{\alpha} \\
& V_{s}=0.2^{\alpha}
\end{aligned}
$$

1054 where the exponent $\alpha=0.7$ makes the value function a concave function of reward amounts, 1055 similar to the fitting analysis of the fluorometry data (Figure 4C). Using $\alpha=1$ (i.e. a linear 1056 function) did not change the results. 
1058 Ourcome1 state, or Water/No-water states (W and N, respectively) represent when the animal 1059 noticed the presence or absence of reward, respectively, but not the amount of reward. The value

1060 of a W (Water) state was defined by the average value of the next states. At the BIG side, 1061

$$
V_{W B}=\left(V_{b}+V_{m}\right) / 2
$$

1062

1063 whereas at the SMALL side,

1064

$$
V_{W S}=\left(V_{m}+V_{s}\right) / 2
$$

1065

The values of $\mathrm{N}$ (No-water) states at the BIG and SMALL side are zero,

1066

1067

1068

1069

1070

1071

1072

1073

1074

1075

1076

1077

1078

1079

1080

1081

1082

1083

1084

1085

1086

$$
\begin{aligned}
& V_{N B}=0 \\
& V_{N S}=0
\end{aligned}
$$

WaterPort1 and WaterPort2 states represent when the animal entered and stayed in the water port, respectively. The state value was obtained separately for the BIG and SMALL side. The value of choosing the BIG and SMALL sides is given by weighted sum of the values of the next states $\left(V_{W B}, V_{N B}, V_{W S}, V_{N S}\right)$. The probabilities of transiting to the $\mathrm{W}$ and $\mathrm{N}$ states are given by the probability of receiving a reward given the choice (BIG or SMALL). As discussed above, these probabilities are given by $f_{B}$ and $f_{S}$, respectively. Thus,

$$
\begin{aligned}
& V_{B}=f_{B} \cdot V_{W B} \\
& V_{S}=f_{S} \cdot V_{W S}
\end{aligned}
$$

We considered two successive states for WaterPort states to reflect a relatively long duration before receiving feedback/outcome. The two successive states had the same state values.

SubOdor1 and SubOdor2 states represent when the animal obtained a subjective odor (O') and before making a choice. The model chooses the BIG or SMALL side with the probability of $P_{B}$ and $P_{S}$, respectively, as defined above. Therefore, the state value of WaterPort1 and WaterPort2 was defined by the weighted sum of the values of the next states $\left(V_{B}\right.$ and $\left.V_{S}\right)$,

$$
V_{O^{\prime}}=P_{B} V_{B}+P_{S} V_{S}
$$

The two successive states had the same state values. 
1087 OdorOn state represents when the animal recognized the presentation of an odor but before

1088

1089

1090

1091

1092

1093

1094

1095

1096

1097

1098

1099

1100

1101

1102

1103

1104

1105

1106

1107

1108

1109

1110

1111

1112

1113

1114

1115

1116

1117 recognizing the identity of that odor. The state value of the OdorOn state is defined by the weighted sum of the values of the next states (SubOdor1).

ITI state represents when the animal is in the inter-trial interval (i.e. before odor presentation). The value of ITI state was set to zero.

TD errors

After obtaining state values at each state, we then computed TD errors using a standard definition of TD error which is the difference between the state values at consecutive time points plus received rewards at each time step (Sutton and Barto, 1987). For simplicity, a discounting factor was set to 1 (no discounting).

\section{Invalid trials}

We also tested the effect of including invalid trials. At water acquisition, we included failures ( $20 \%$ of trials, value 0$)$ where a mouse did not fulfil the requirement of odor poke duration (short odor poke), but did indicate a choice. At an odor port, failures resulted from multiple pokes of odor port (4\% of trials), and a short odor poke (14\% of trials). Values for these failures were set to 0 . Existence or omission of these failures in models did not change the conclusion.

\section{Randomization, blinding, and data exclusion}

Chemicals were randomly assigned to an odor cue. Trial types (odors) were pseudorandomized in a block. Session types were pseudorandomized in a recording schedule. Animals were randomly assigned to a recording location. The experimenter did not know location of recording until the recording schedule was completed. No animals were excluded from the study: all analysis includes data from all animals. No trials were excluded from statistical analyses. To visualize average activity pattern in a stretched time-window, outlier trials (maximum, minimum or average activity of a trial is outside of $3 \times$ standard deviation of maximum, minimum or average activity of all the trials) were excluded.

\section{Statistical analyses}


1118 Data analysis was performed using custom software written in Matlab (MathWorks, Natick, MA, 1119 USA). All code used for analysis is available on request. All statistical tests were two-sided. For 1120 statistical comparisons of the mean, we used one-way ANOVA and two-sample Student's t tests, 1121 unless otherwise noted. Paired t tests were conducted when the same mouse's neural activity was 1122 being compared across different conditions or different time windows. The significance level 1123 was corrected for multiple comparisons using Holm-Sidak's tests unless otherwise indicated. All 1124 error bars in the figures are s.e.m. In boxplots, the edges of the boxes are the 25 th and 75 th 1125 percentiles ( $\mathrm{q} 1$ and $\mathrm{q} 3$, respectively), and the whiskers extend to the most extreme data points not 1126 considered outliers. Points are drawn as outliers if they are larger than $\mathrm{q} 3+1.5 \times(\mathrm{q} 3-\mathrm{q} 1)$ or q1$11271.5 \times(\mathrm{q} 3-\mathrm{q} 1)$. Individual data points were overlaid on boxplots to compare striatal areas. 


\section{Author Contributions:}

1131 ITK and MWU designed experiments and collected and analyzed data. HM performed pilot experiments.

1132 The results were discussed and interpreted by ITK, NU and MWU. ITK, NU and MWU wrote the paper.

\section{Acknowledgments}

1138 We thank Ju Tian, William Menegas, HyungGoo Kim and Takahiro Yamaguchi for technical assistance, 1139 Kristen Fang, Grace Chang, Melissa Yamada and Sakura Ikeda for assistance in animal training and 1140 histology, and all lab members for discussion. We also thank V. Jayaraman, R. Kerr, D. Kim, L. Looper, 1141 and K. Svoboda from the GENIE Project, Janelia Farm Research Campus, Howard Hughes Medical 1142 Institute for AAV-FLEX-GCaMP7f. This work was supported by National Institute of Mental Health 1143 R01MH095953, R01MH101207, R01MH110404, R01NS108740 (NU); and Japan Society for the 1144 Promotion of Science, Japan Science and Technology Agency (HM, ITK).

1147 Declaration of Interests

1149 The authors declare no competing interests. 


\section{REFERENCE}

1154 Adler, W.T., and Ma, W.J. (2018). Limitations of Proposed Signatures of Bayesian Confidence.

1155 Neural Comput. 30, 3327-3354.

Bäckman, C.M., Malik, N., Zhang, Y., Shan, L., Grinberg, A., Hoffer, B.J., Westphal, H., and Tomac, A.C. (2006). Characterization of a mouse strain expressing Cre recombinase from the 3' untranslated region of the dopamine transporter locus. Genes. N. Y. N 2000 44, 383-390.

Balleine, B.W., and Dickinson, A. (1998). Goal-directed instrumental action: contingency and incentive learning and their cortical substrates. Neuropharmacology 37, 407-419. $35,48-69$.

Bayer, H.M., and Glimcher, P.W. (2005). Midbrain dopamine neurons encode a quantitative reward prediction error signal. Neuron 47, 129-141. food reward and reward-predictive stimuli evoke different patterns of phasic dopamine signaling throughout the striatum. Eur. J. Neurosci. 34, 1997-2006. Produce a Conditioned Inhibitor. J. Neurosci. 38, 8822-8830. motor cortex. Neuron 94, 866-879.

1174 Clark, J.J., Hollon, N.G., and Phillips, P.E. (2012). Pavlovian valuation systems in learning and 1175 decision making. Curr. Opin. Neurobiol. 22, 1054-1061.

1176 Cohen, J.Y., Haesler, S., Vong, L., Lowell, B.B., and Uchida, N. (2012). Neuron-type-specific 1177 signals for reward and punishment in the ventral tegmental area. Nature 482, 85-88.

1178 Cox, J., and Witten, I.B. (2019). Striatal circuits for reward learning and decision-making. Nat. 1179 Rev. Neurosci. 20, 482-494.

1180 Dabney, W., Kurth-Nelson, Z., Uchida, N., Starkweather, C.K., Hassabis, D., Munos, R., and Nature 1-5. 
Daw, N.D., Niv, Y., and Dayan, P. (2005). Uncertainty-based competition between prefrontal and dorsolateral striatal systems for behavioral control. Nat. Neurosci. 8, 1704-1711.

Dayan, P., and Berridge, K.C. (2014). Model-based and model-free Pavlovian reward learning:

1190 Dezfouli, A., and Balleine, B.W. (2012). Habits, action sequences and reinforcement learning. 1191 Eur. J. Neurosci. 35, 1036-1051.

1192 Dickinson, A., and Weiskrantz, L. (1985). Actions and habits: the development of behavioural 1193 autonomy. Philos. Trans. R. Soc. Lond. B Biol. Sci. 308, 67-78.

1194 Dolan, R.J., and Dayan, P. (2013). Goals and Habits in the Brain. Neuron 80, 312-325.

1195 Engelhard, B., Finkelstein, J., Cox, J., Fleming, W., Jang, H.J., Ornelas, S., Koay, S.A., Thiberge, 1196 S.Y., Daw, N.D., Tank, D.W., et al. (2019). Specialized coding of sensory, motor and cognitive 1197 variables in VTA dopamine neurons. Nature 570, 509-513. response function for reward prediction error. Nat. Neurosci. 19, 479-486.

1200 Evans, R.C., Zhu, M., and Khaliq, Z.M. (2017). Dopamine inhibition differentially controls 1201 excitability of substantia nigra dopamine neuron subpopulations through T-type calcium 1202 channels. J. Neurosci. 37, 3704-3720.

1203 Farassat, N., Costa, K.M., Stojanovic, S., Albert, S., Kovacheva, L., Shin, J., Egger, R., Somayaji, 1204 M., Duvarci, S., and Schneider, G. (2019). In vivo functional diversity of midbrain dopamine 1205 neurons within identified axonal projections. Elife 8.

1206 Gerfen, C.R., and Surmeier, D.J. (2011). Modulation of Striatal Projection Systems by Dopamine. 1207 Annu. Rev. Neurosci. 34, 441-466.

1208 Graybiel, A.M. (2008). Habits, Rituals, and the Evaluative Brain. Annu. Rev. Neurosci. 31, 3591209387.

1210 Green, D.M., and Swets, J.A. (1966). Signal detection theory and psychophysics (Wiley New 1211 York).

1212 Hangya, B., Sanders, J.I., and Kepecs, A. (2016). A Mathematical Framework for Statistical 1213 Decision Confidence. Neural Comput. 28, 1840-1858.

1214 Hart, A.S., Rutledge, R.B., Glimcher, P.W., and Phillips, P.E. (2014). Phasic dopamine release in 1215 the rat nucleus accumbens symmetrically encodes a reward prediction error term. J. Neurosci. $121634,698-704$. 
1217 Herrnstein, R.J. (1961). Relative and absolute strength of responses as a function of frequency 1218 of reinforcement.

1219 Hikosaka, O., Rand, M.K., Miyachi, S., and Miyashita, K. (1995). Learning of sequential

1220 movements in the monkey: process of learning and retention of memory. J. Neurophysiol. 74,

1221 1652-1661.

1222 Hirokawa, J., Vaughan, A., Masset, P., Ott, T., and Kepecs, A. (2019). Frontal cortex neuron types 1223 categorically encode single decision variables. Nature 576, 446-451.

1224 Hong, S., Jhou, T.C., Smith, M., Saleem, K.S., and Hikosaka, O. (2011). Negative reward signals 1225 from the lateral habenula to dopamine neurons are mediated by rostromedial tegmental 1226 nucleus in primates. J. Neurosci. 31, 11457-11471.

1227 Howe, M.W., and Dombeck, D.A. (2016). Rapid signalling in distinct dopaminergic axons during 1228 locomotion and reward. Nature 535, 505-510.

1229 Hunnicutt, B.J., Jongbloets, B.C., Birdsong, W.T., Gertz, K.J., Zhong, H., and Mao, T. (2016). A 1230 comprehensive excitatory input map of the striatum reveals novel functional organization. ELife 12315.

1232 Ilango, A., Kesner, A.J., Keller, K.L., Stuber, G.D., Bonci, A., and Ikemoto, S. (2014). Similar Roles 1233 of Substantia Nigra and Ventral Tegmental Dopamine Neurons in Reward and Aversion. J.

1234 Neurosci. 34, 817-822.

1235 Jhou, T.C., Geisler, S., Marinelli, M., Degarmo, B.A., and Zahm, D.S. (2009a). The mesopontine 1236 rostromedial tegmental nucleus: a structure targeted by the lateral habenula that projects to 1237 the ventral tegmental area of Tsai and substantia nigra compacta. J. Comp. Neurol. 513, 5661238596.

1239 Jhou, T.C., Fields, H.L., Baxter, M.G., Saper, C.B., and Holland, P.C. (2009b). The rostromedial 1240 tegmental nucleus (RMTg), a GABAergic afferent to midbrain dopamine neurons, encodes 1241 aversive stimuli and inhibits motor responses. Neuron 61, 786-800.

1242 de Jong, J.W., Afjei, S.A., Pollak Dorocic, I., Peck, J.R., Liu, C., Kim, C.K., Tian, L., Deisseroth, K., 1243 and Lammel, S. (2019). A Neural Circuit Mechanism for Encoding Aversive Stimuli in the 1244 Mesolimbic Dopamine System. Neuron 101, 133-151.e7.

1245 Kamin, L.J. (1969). Predictability, surprise, attention and conditioning. Punishm. Aversive Behav.

1246 Keiflin, R., Pribut, H.J., Shah, N.B., and Janak, P.H. (2019). Ventral Tegmental Dopamine Neurons 1247 Participate in Reward Identity Predictions. Curr. Biol. 29, 93-103.e3.

1248 Kepecs, A., Uchida, N., Zariwala, H.A., and Mainen, Z.F. (2008). Neural correlates, computation 1249 and behavioural impact of decision confidence. Nature 455, 227-231. 
1250 Kim, H.F., Ghazizadeh, A., and Hikosaka, O. (2015). Dopamine Neurons Encoding Long-Term 1251 Memory of Object Value for Habitual Behavior. Cell 163, 1165-1175.

1252 Kudo, Y., Akita, K., Nakamura, T., Ogura, A., Makino, T., Tamagawa, A., Ozaki, K., and Miyakawa, 1253 A. (1992). A single optical fiber fluorometric device for measurement of intracellular Ca2+ 1254 concentration: its application to hippocampal neurons in vitro and in vivo. Neuroscience 50, 1255 619-625.

1256 Lak, A., Nomoto, K., Keramati, M., Sakagami, M., and Kepecs, A. (2017). Midbrain dopamine neurons signal belief in choice accuracy during a perceptual decision. Curr. Biol. 27, 821-832.

Lak, A., Hueske, E., Hirokawa, J., Masset, P., Ott, T., Urai, A.E., Donner, T.H., Carandini, M., Tonegawa, S., Uchida, N., et al. (2020a). Reinforcement biases subsequent perceptual decisions when confidence is low, a widespread behavioral phenomenon. ELife 9, e49834. Harris, K.D., and Carandini, M. (2020b). Dopaminergic and prefrontal basis of learning from sensory confidence and reward value. Neuron 105, 700-711. 773.

1267 Lee, K., Claar, L.D., Hachisuka, A., Bakhurin, K.I., Nguyen, J., Trott, J.M., Gill, J.L., and 1268 Masmanidis, S.C. (2020). Temporally restricted dopaminergic control of reward-conditioned 1269 movements. Nat. Neurosci. 23, 209-216.

1270 Lerner, T.N., Shilyansky, C., Davidson, T.J., Evans, K.E., Beier, K.T., Zalocusky, K.A., Crow, A.K., 1271 Malenka, R.C., Luo, L., Tomer, R., et al. (2015). Intact-Brain Analyses Reveal Distinct Information 1272 Carried by SNc Dopamine Subcircuits. Cell 162, 635-647.

1273 Li, H., Vento, P.J., Parrilla-Carrero, J., Pullmann, D., Chao, Y.S., Eid, M., and Jhou, T.C. (2019). 1274 Three Rostromedial Tegmental Afferents Drive Triply Dissociable Aspects of Punishment 1275 Learning and Aversive Valence Encoding. Neuron 104, 987-999.e4.

1276 Lloyd, K., and Dayan, P. (2016). Safety out of control: dopamine and defence. Behav. Brain 1277 Funct. BBF 12, 15.

1278 Madisen, L., Zwingman, T.A., Sunkin, S.M., Oh, S.W., Zariwala, H.A., Gu, H., Ng, L.L., Palmiter, 1279 R.D., Hawrylycz, M.J., Jones, A.R., et al. (2010). A robust and high-throughput Cre reporting and 1280 characterization system for the whole mouse brain. Nat. Neurosci. 13, 133-140.

1281 Malvaez, M., and Wassum, K.M. (2018). Regulation of habit formation in the dorsal striatum. 1282 Curr. Opin. Behav. Sci. 20, 67-74. 
1283 Matsumoto, M., and Hikosaka, O. (2007). Lateral habenula as a source of negative reward 1284 signals in dopamine neurons. Nature 447, 1111-1115.

1285 Matsumoto, M., and Hikosaka, O. (2009). Two types of dopamine neuron distinctly convey 1286 positive and negative motivational signals. Nature 459, 837-841.

1287 Matsuzaka, Y., Picard, N., and Strick, P.L. (2007). Skill Representation in the Primary Motor 1288 Cortex After Long-Term Practice. J. Neurophysiol. 97, 1819-1832.

1289 Menegas, W., Bergan, J.F., Ogawa, S.K., Isogai, Y., Umadevi Venkataraju, K., Osten, P., Uchida, 1290 N., and Watabe-Uchida, M. (2015). Dopamine neurons projecting to the posterior striatum 1291 form an anatomically distinct subclass. ELife 4, e10032.

1292 Menegas, W., Babayan, B.M., Uchida, N., and Watabe-Uchida, M. (2017). Opposite initialization 1293 to novel cues in dopamine signaling in ventral and posterior striatum in mice. ELife 6.

1294 Menegas, W., Akiti, K., Amo, R., Uchida, N., and Watabe-Uchida, M. (2018). Dopamine neurons 1295 projecting to the posterior striatum reinforce avoidance of threatening stimuli. Nat. Neurosci. $129621,1421-1430$.

Miller, K.J., Shenhav, A., and Ludvig, E.A. (2019). Habits without values. Psychol. Rev. 126, 2921298311.

1299 Montague, P.R., Dayan, P., and Sejnowski, T.J. (1996). A framework for mesencephalic 1300 dopamine systems based on predictive Hebbian learning. J. Neurosci. 16, 1936-1947.

1301 Morris, A., and Cushman, F. (2019). Model-Free RL or Action Sequences? Front. Psychol. 10.

1302 O’Doherty, J., Dayan, P., Schultz, J., Deichmann, R., Friston, K., and Dolan, R.J. (2004).

1303 Dissociable Roles of Ventral and Dorsal Striatum in Instrumental Conditioning. Science 304, 1304 452-454.

1305 Ölveczky, B.P. (2011). Motoring ahead with rodents. Curr. Opin. Neurobiol. 21, 571-578.

1306 Oyama, K., Hernádi, I., lijima, T., and Tsutsui, K.-I. (2010). Reward Prediction Error Coding in 1307 Dorsal Striatal Neurons. J. Neurosci. 30, 11447-11457.

1308 Park, I.M., Meister, M.L.R., Huk, A.C., and Pillow, J.W. (2014). Encoding and decoding in parietal 1309 cortex during sensorimotor decision-making. Nat. Neurosci. 17, 1395-1403. depends on striatal target. Nat. Neurosci. 19, 845-854.

1313 Paxinos, G., and Franklin, K.B.J. (2019). Paxinos and Franklin's the Mouse Brain in Stereotaxic 1314 Coordinates (Academic Press). 
Pearce, J.M., and Hall, G. (1980). A model for Pavlovian learning: variations in the effectiveness of conditioned but not of unconditioned stimuli. Psychol. Rev. 87, 532.

1317 Rangel, A., Camerer, C., and Montague, P.R. (2008). A framework for studying the neurobiology 1318 of value-based decision making. Nat. Rev. Neurosci. 9, 545-556.

1319 Rausch, M., and Zehetleitner, M. (2019). The folded X-pattern is not necessarily a statistical 1320 signature of decision confidence. PLOS Comput. Biol. 15, e1007456.

1321 Rescorla, R.A., and Wagner, A.R. (1972). A theory of Pavlovian conditioning: Variations in the 1322 effectiveness of reinforcement and nonreinforcement. Class. Cond. II Curr. Res. Theory 2, 64132399.

1324 Robbins, T.W., and Costa, R.M. (2017). Habits. Curr. Biol. 27, R1200-R1206.

1325 Rorie, A.E., Gao, J., McClelland, J.L., and Newsome, W.T. (2010). Integration of Sensory and 1326 Reward Information during Perceptual Decision-Making in Lateral Intraparietal Cortex (LIP) of 1327 the Macaque Monkey. PLOS ONE 5, e9308.

1328 Sajad, A., Godlove, D.C., and Schall, J.D. (2019). Cortical microcircuitry of performance 1329 monitoring. Nat. Neurosci. 22, 265-274. in Corticobasal Ganglia Loops. Ann. N. Y. Acad. Sci. 1104, 213-228.

Saunders, B.T., Richard, J.M., Margolis, E.B., and Janak, P.H. (2018). Dopamine neurons create Pavlovian conditioned stimuli with circuit-defined motivational properties. Nat. Neurosci. 21, 1334 1072-1083.

1335 Schultz, W., Dayan, P., and Montague, P.R. (1997). A neural substrate of prediction and reward. 1336 Science 275, 1593-1599.

1337 da Silva, J.A., Tecuapetla, F., Paixão, V., and Costa, R.M. (2018). Dopamine neuron activity 1338 before action initiation gates and invigorates future movements. Nature 554, 244-248.

1339 Smith, K.S., and Graybiel, A.M. (2016). Habit formation. Dialogues Clin. Neurosci. 18, 33-43.

1340 Stuphorn, V., Taylor, T.L., and Schall, J.D. (2000). Performance monitoring by the supplementary 1341 eye field. Nature 408, 857-860.

1342 Suri, R.E., and Schultz, W. (1999). A neural network model with dopamine-like reinforcement 1343 signal that learns a spatial delayed response task. Neuroscience 91, 871-890.

1344 Sutton, R.S. (1988). Learning to predict by the methods of temporal differences. Mach. Learn. 3, 1345 9-44. 
Sutton, R.S., and Barto, A.G. (1987). A temporal-difference model of classical conditioning. In Proceedings of the Ninth Annual Conference of the Cognitive Science Society, (Seattle, WA), pp. 355-378.

Sutton, R.S., and Barto, A.G. (2011). Reinforcement learning: An introduction.

1350 Sutton, R.S., and Barto, A.G. (2018). Reinforcement Learning, second edition: An Introduction 1351 (MIT Press).

1352 Thorndike, E.L. (1932). The fundamentals of learning (New York, NY, US: Teachers College 1353 Bureau of Publications).

1354 Tian, J., Huang, R., Cohen, J.Y., Osakada, F., Kobak, D., Machens, C.K., Callaway, E.M., Uchida, N., 1355 and Watabe-Uchida, M. (2016). Distributed and Mixed Information in Monosynaptic Inputs to 1356 Dopamine Neurons. Neuron 91, 1374-1389.

1357 Uchida, N., and Mainen, Z.F. (2003). Speed and accuracy of olfactory discrimination in the rat. 1358 Nat. Neurosci. 6, 1224-1229.

1359 Wang, A.Y., Miura, K., and Uchida, N. (2013). The dorsomedial striatum encodes net expected 1360 return, critical for energizing performance vigor. Nat. Neurosci. 16, 639-647.

1361 Watabe-Uchida, M., and Uchida, N. (2018). Multiple dopamine systems: Weal and woe of 1362 dopamine. In Cold Spring Harbor Symposia on Quantitative Biology, (Cold Spring Harbor 1363 Laboratory Press), pp. 83-95.

1364 Yetnikoff, L., Lavezzi, H.N., Reichard, R.A., and Zahm, D.S. (2014). An update on the connections 1365 of the ventral mesencephalic dopaminergic complex. Neuroscience 282, 23-48.

1366 Yin, H.H., Knowlton, B.J., and Balleine, B.W. (2004). Lesions of dorsolateral striatum preserve 1367 outcome expectancy but disrupt habit formation in instrumental learning. Eur. J. Neurosci. 19, 1368 181-189.

1369 Yin, H.H., Ostlund, S.B., Knowlton, B.J., and Balleine, B.W. (2005). The role of the dorsomedial 1370 striatum in instrumental conditioning. Eur. J. Neurosci. 22, 513-523. 
bioRxiv preprint doi: https://doi.org/10.1101/2020.08.22.262972; this version posted August 22, 2020. The copyright holder for this preprint (which was not certified by peer review) is the author/funder, who has granted bioRxiv a license to display the preprint in perpetuity. It is made available under aCC-BY-NC-ND 4.0 International license.

Figure 1
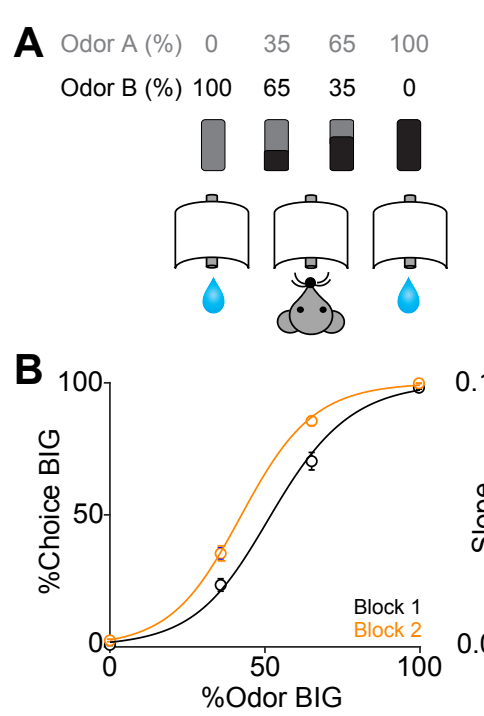

D
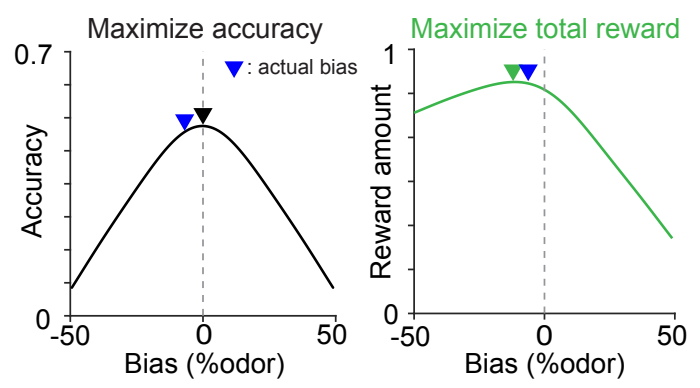

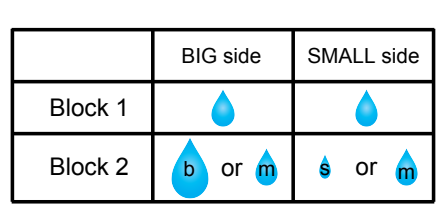

0.14
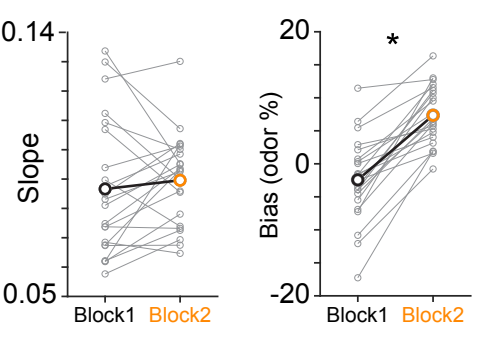

Minimize variance

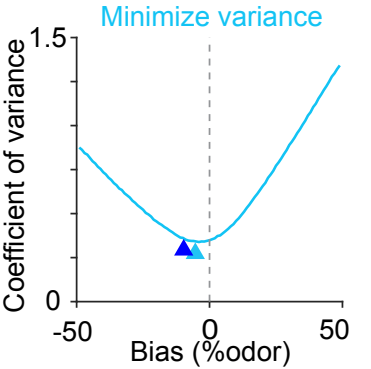

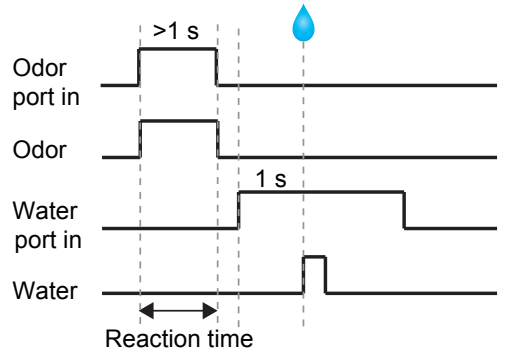

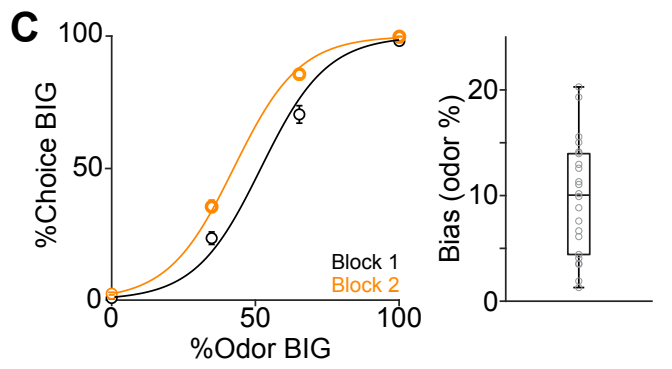

E

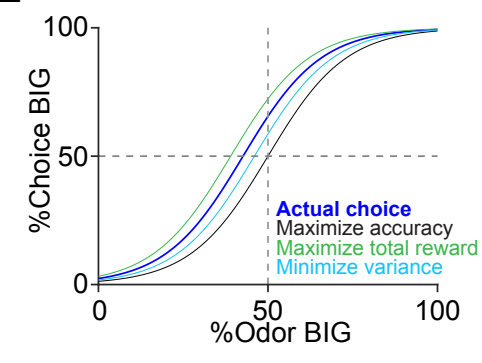

F
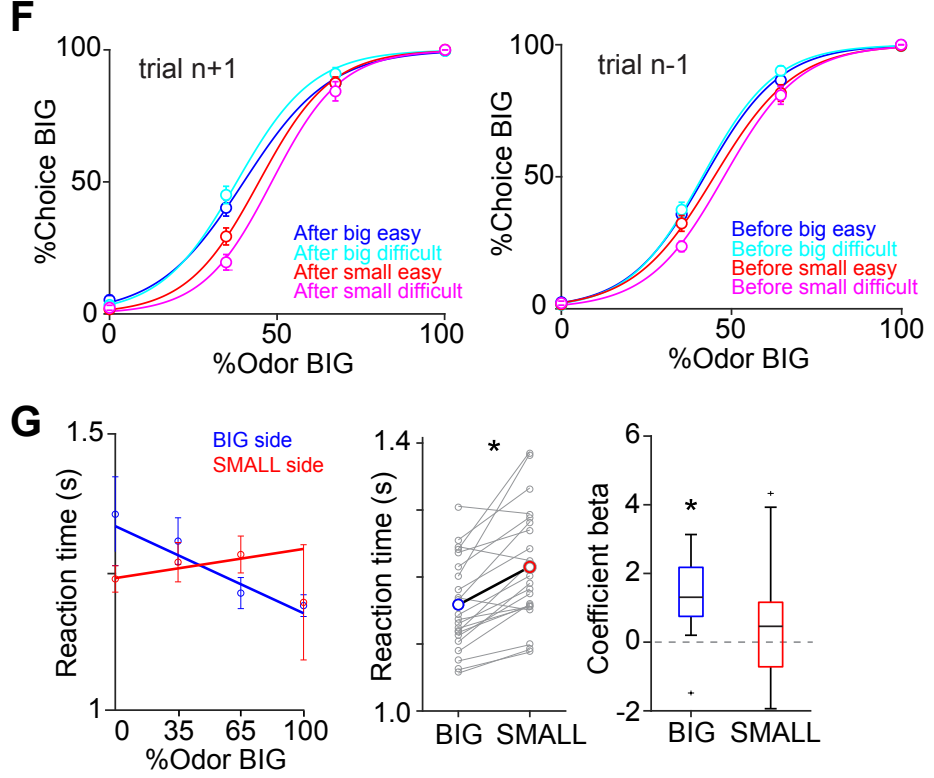

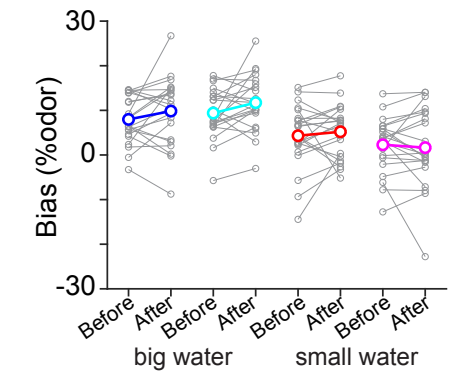

big water small water 
Figure 1. Perceptual choice paradigm with probabilistic reward conditions (A) A mouse discriminated a dominant odor in odor mixtures that indicates water availability in either the left or right water port. Correct choice was rewarded by a drop of water. In each session, an equal amount of water was assigned at both water ports in the first block, and in the second block, $\mathrm{big} / \mathrm{medium}$ water (50\% 50\%, randomized) was assigned at one water port (BIG side) and medium/small water (50\% 50\%, randomized) was assigned at another port (SMALL side). The BIG or SMALL side was assigned to a left or right water port in a pseudorandom order across sessions. (B) Left, $\%$ of choice of the BIG side in block 1 and 2 (mean \pm SEM) and the average psychometric curve for each block. Center, slope of the psychometric curve. Right, choice bias at 50/50 choice, expressed as 50 - odor (\%). (C) Left, \% of choice of the BIG side in block 1 and 2 $($ mean \pm SEM) and the average psychometric curve with a fixed slope across blocks. Right, all the animals showed choice bias toward BIG side in block 2 compared to block 1 . The choice bias was expressed by a lateral shift of a psychometric curve with a fixed slope across blocks. (D) Average reward amounts, accuracy, and coefficients of variance were examined with different levels of choice bias with a fixed slope (average slope of all animals). (E) Optimal choice patterns with different strategies in D (bias $-11,0$, and -4 , respectively) and the actual average choice pattern (mean bias -7.3). (F) Trial-by-trial choice updating was examined by comparing choice bias before (center, trial $n-1)$ and after (left, trial $n+1)$ specific trial types. Choice updating in one trial was not significant for reward acquisition of either small or big water in easy or difficult trials (right). (G) Left, animal's reaction time was modulated by odor types. Center, for easy trials (pure odors, correct choice), reaction time was shorter when animals chose the BIG side $\left(\mathrm{p}=2.7 \times 10^{-5}\right)$. Right, the reaction time was negatively correlated with sensory evidence for choice of the BIG side $\left(\mathrm{p}=1.2 \times 10^{-4}\right)$, whereas the modulation was not significant for choice of the SMALL side $(\mathrm{p}=0.13) . \mathrm{n}=22$ animals. 
bioRxiv preprint doi: https://doi.org/10.1101/2020.08.22.262972; this version posted August 22, 2020. The copyright holder for this preprint (which was not certified by peer review) is the author/funder, who has granted bioRxiv a license to display the preprint in perpetuity. It is made available under aCC-BY-NC-ND 4.0 International license.

\section{Figure 2}

A

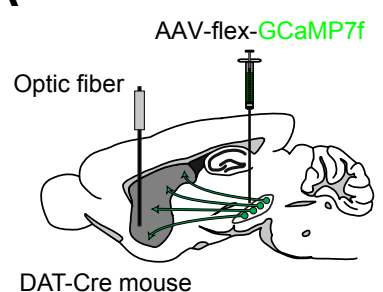

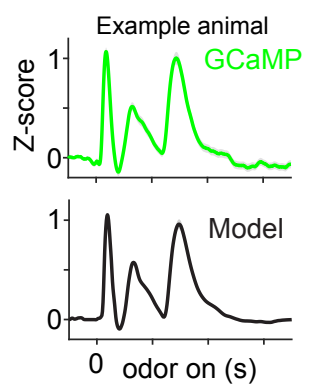

C

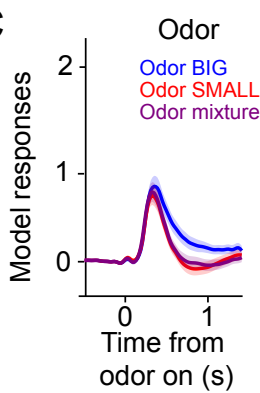

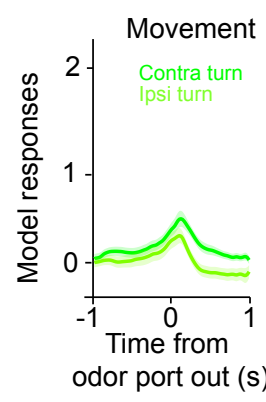

B
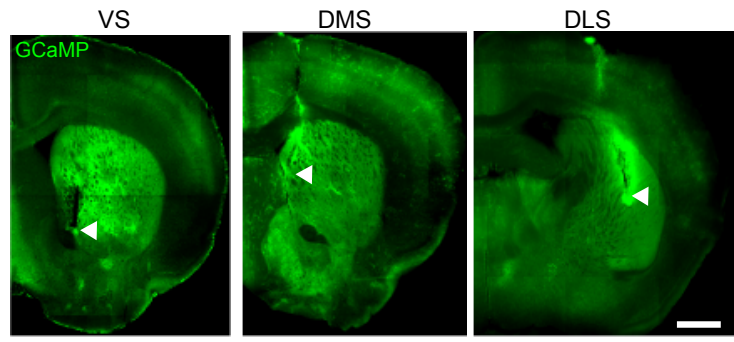

Water
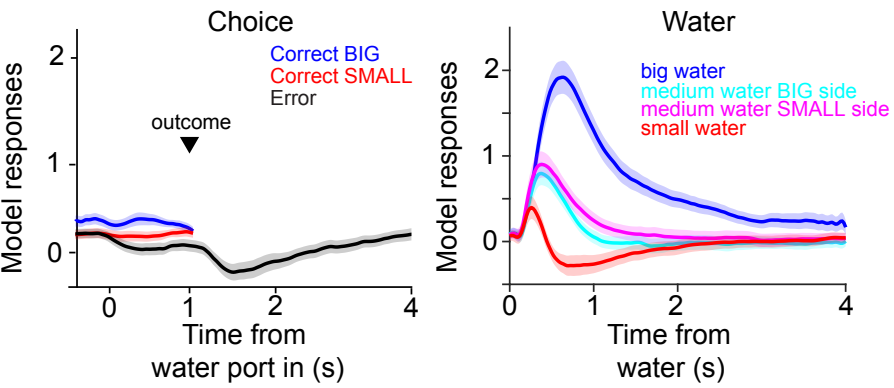

E
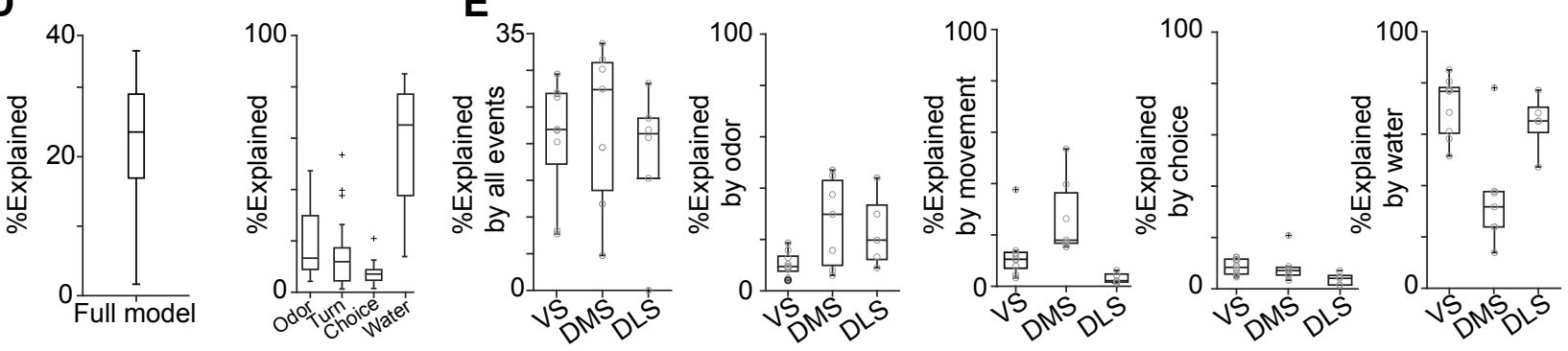

F
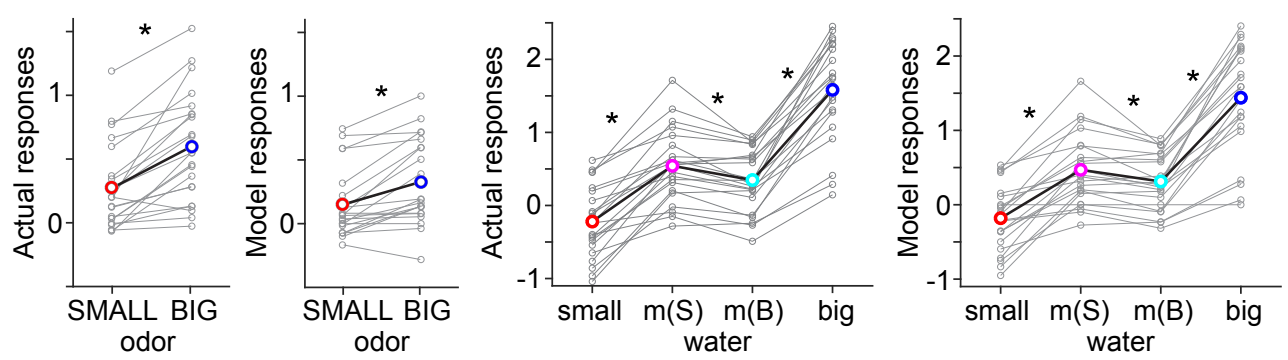

G

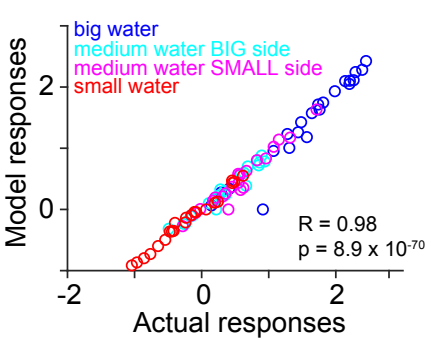


Figure 2. Dopamine axons in the striatum show characteristics of RPE (A) AAV-flexGCaMP7f was injected in VTA and SNc, and dopamine axon activity was measured with an optic fiber inserted in the striatum. Right top, dopamine axon activity in all the valid trials (an animal chose an either water port after wait for the required stay time) in an example animal, aligned at odor onset (mean \pm SEM). Right bottom, a fitted model of the same animal (mean \pm SEM). (B) Location of an optic fiber in example animals. Arrow heads, tips of fibers. Green, GCaMP7f. Bar = $1 \mathrm{~mm}(\mathrm{C})$ Odor-, movement-, choice-, and water-locked components in the model of all the animals (mean $\pm \mathrm{SEM}$ ). (D) Contribution of each component in the model was measured by reduction of deviance in the full model compared to a reduced model excluding the component. (E) Contribution of each component in the model in each animal group. (F) Left, comparison of dopamine axon responses to an odor cue that instructs to choose BIG and SMALL side in easy trials (pure odor, correct choice, $-1-0 \mathrm{~s}$ before odor port out). $\mathrm{p}=5.0 \times 10^{-6}$ for actual signals and $\mathrm{p}=7.4 \times 10^{-5}$ for models. Right, comparison of dopamine axon responses to different sizes of water (big versus medium water with BIG expectation, and medium versus small water with SMALL expectation) and to medium water with different expectation (BIG versus SMALL expectation) $\left(0.3-1.3 \mathrm{~s}\right.$ after water onset). $\mathrm{p}=1.2 \times 10^{-11}, \mathrm{p}=3.8 \times 10^{-9}$ and $\mathrm{p}=3.9 \times 10^{-4}$, respectively for actual signals, and $\mathrm{p}=1.0 \times 10^{-9}, \mathrm{p}=1.0 \times 10^{-7}$, and $\mathrm{p}=0.0031$, respectively for models. $\mathrm{n}=22$ animals. $\mathrm{m}(\mathrm{B})$, medium water with BIG expectation; $\mathrm{m}(\mathrm{S})$, medium water with SMALL expectation. (G) Comparison between actual dopamine axon responses and model responses to water. 
Figure 3

A
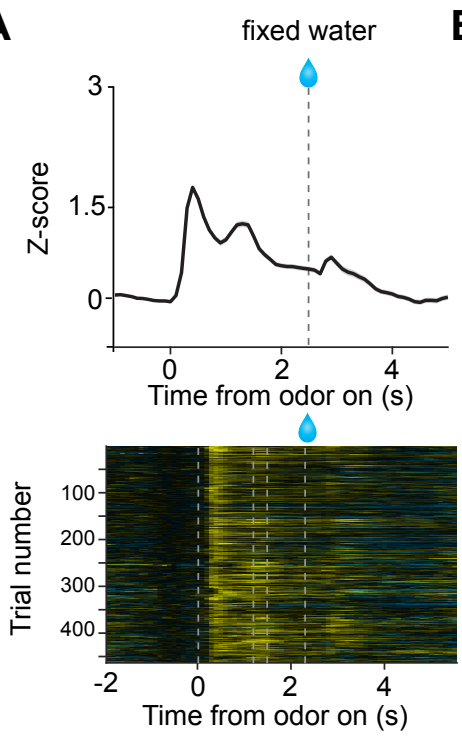

D
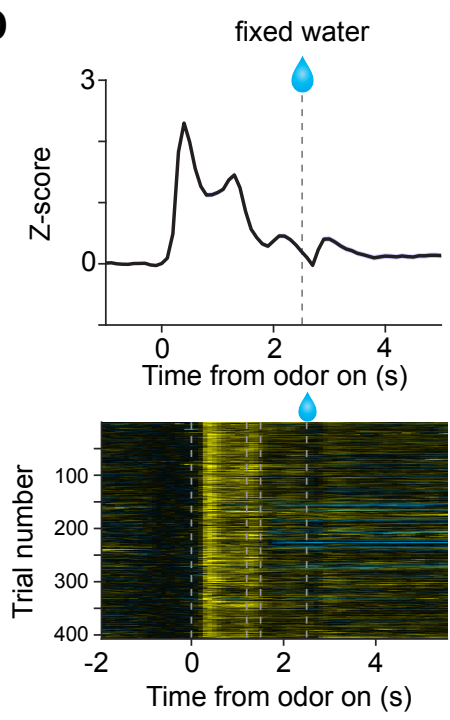

G

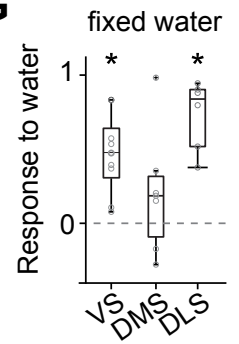

B
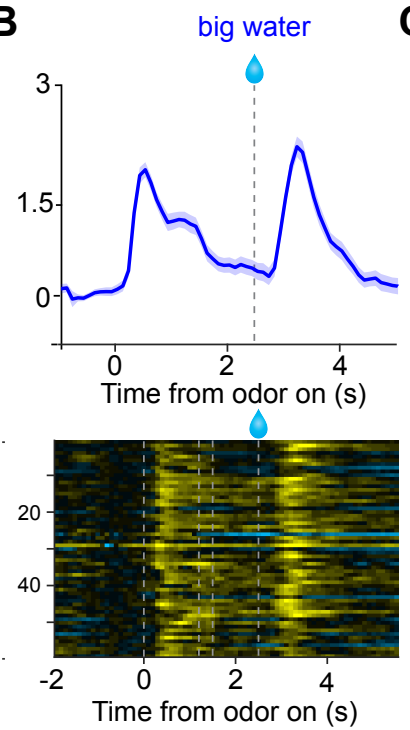

E

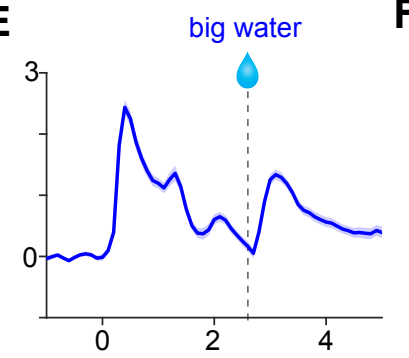

Time from odor on (s)

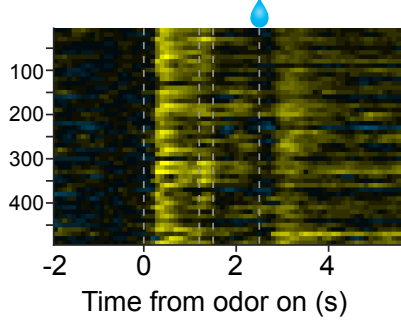

C small water
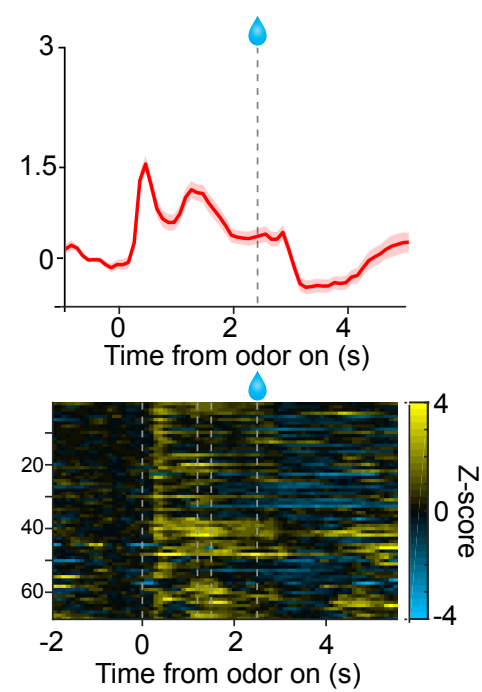

$\mathbf{F}$

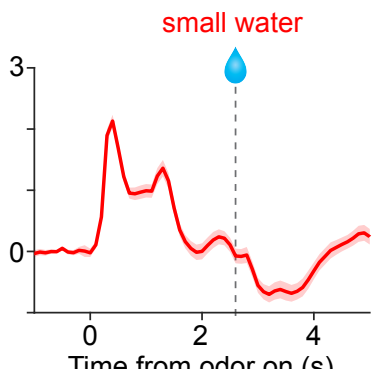

Time from odor on (s)

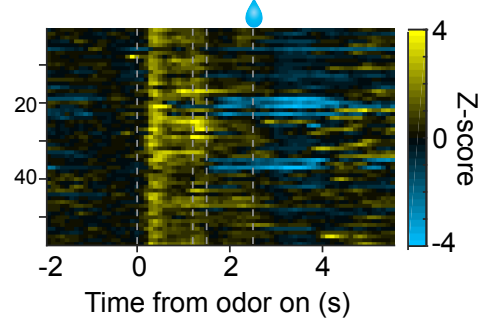

Figure 3. Small responses to fixed amounts of water in dopamine axons in DMS

(A, D) Dopamine axon responses to water in a fixed reward amount task (pure odor, correct choice). (B, E) Dopamine axon responses to a big amount of water in a variable reward amount task (pure odor, correct choice). (C, F) Dopamine axon responses to a small amount of water in a variable reward amount task (pure odor, correct choice). A-C, dopamine axon activity in an example animal; D-F, another example animal. (G) Responses to water (0.3-1.3 s after water onset) were significantly modulated with striatal location ( $\mathrm{p}=0.020$, ANOVA). The water responses were significantly positive in VS $(\mathrm{p}=0.0011)$ and in DLS $\left(\mathrm{p}=6.3 \times 10^{-4}\right)$, but not in DMS $(\mathrm{p}=0.28)$. 
Figure 4

A
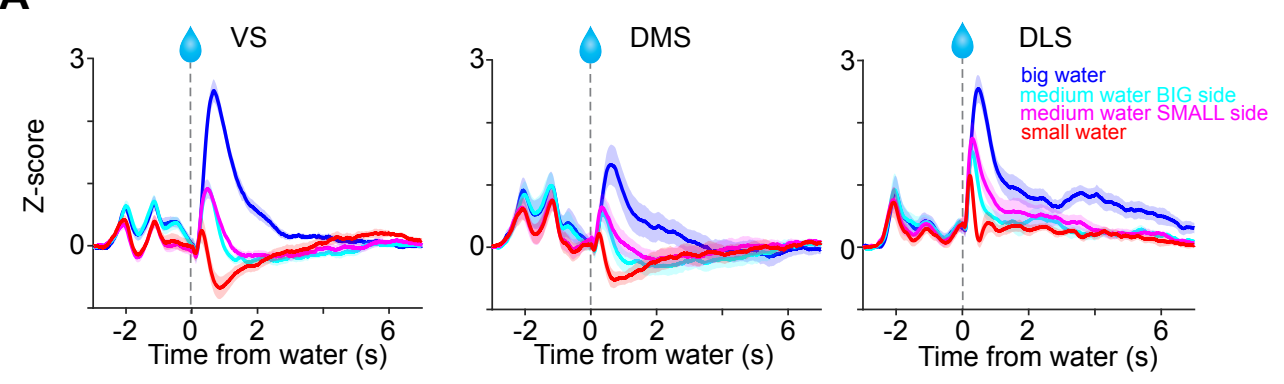

B

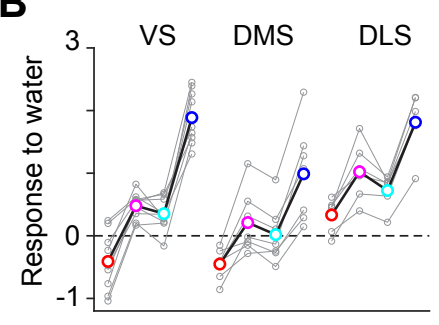

C
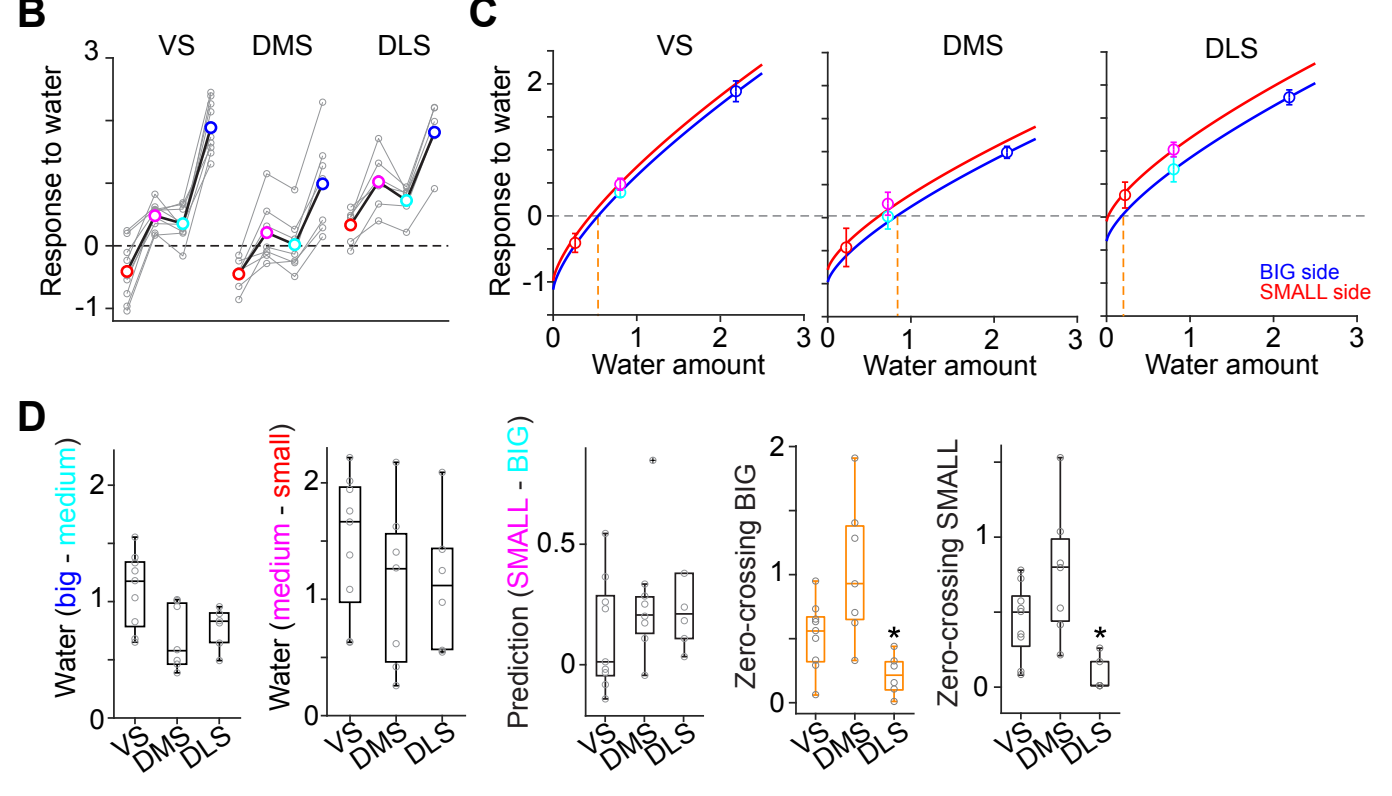

E

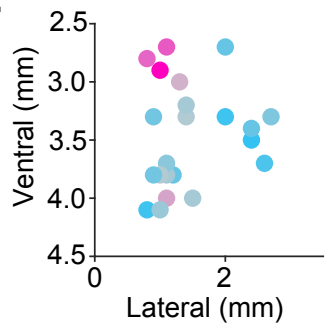

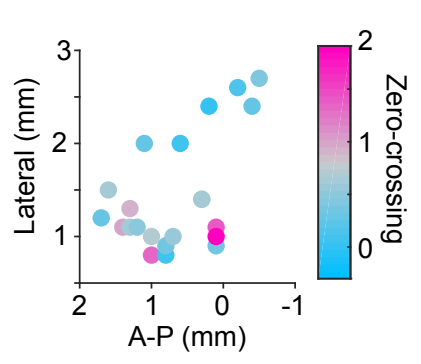

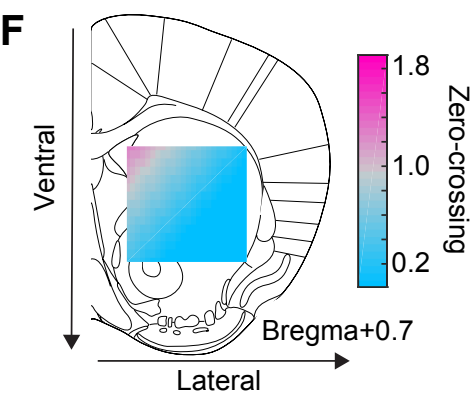


Figure 4. Responses to water in dopamine axons in the striatum (A) Activity patterns per different striatal location, aligned at water onset (mean \pm SEM, $n=9$ for VS, $n=7$ for DMS, $n=6$ for DLS). (B) Average responses to each water condition in each animal grouped by striatal areas. (C) Average response functions of dopamine axons in each striatal area. (D) Comparison of parameters for each animal grouped by striatal areas. "Water big-medium" is responses to big water minus responses to medium water at the BIG side and "Water medium-small" is responses to medium water minus responses to small water at the SMALL side, normalized with difference of water amounts (2.2 minus 0.8 for BIG and 0.8 minus 0.2 for SMALL). "Prediction SMALLBIG" is responses to medium water at SMALL side minus responses to medium water at BIG side. "Zero-crossing BIG" is the water amount when the dopamine response is zero at BIG and side, which was estimated by the obtained response function. "Zero-crossing SMALL" is the water amount when the dopamine response is zero at SMALL side, which was estimated by the obtained response function. Response changes by water amounts (BIG or SMALL) or prediction was not significantly modulated by the striatal areas $(\mathrm{p}=0.011, \mathrm{p}=0.34, \mathrm{p}=0.23$, ANOVA), whereas zero-crossing points (BIG or SMALL) were significantly modulated $(p=0.002, p=0.002$, ANOVA; $p=0.004$, DMS versus DLS for BIG side; $p=0.005$, VS versus DLS; $p=0.003$, DMS versus DLS for SMALL side). (E) Zero-crossing points were plotted along anatomical location in the striatum. Zero-crossing points were correlated with medial-lateral positions $(\mathrm{p}=0.011)$ and with dorsal-ventral positions ( $\mathrm{p}=0.014)$. (F) Zero-crossing points were fitted with recorded location, and the estimated values in the striatal area were overlaid on the atlas for visualization (see Methods). 
Figure 5

A

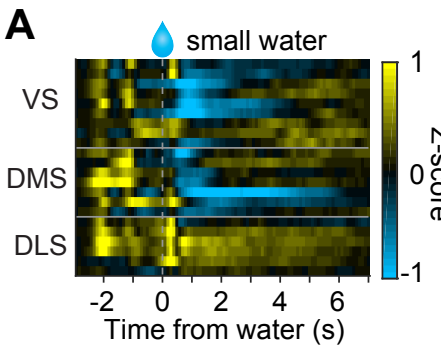

D

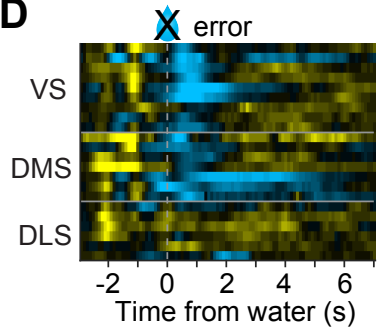

G

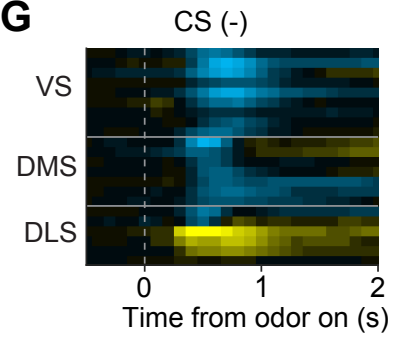

B

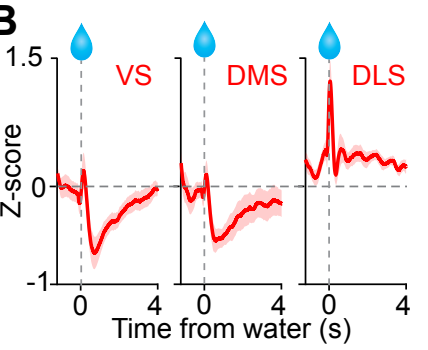

E

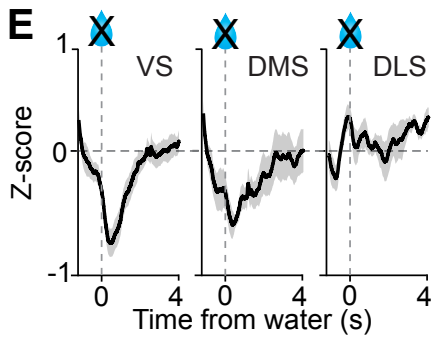

$\mathbf{H}$

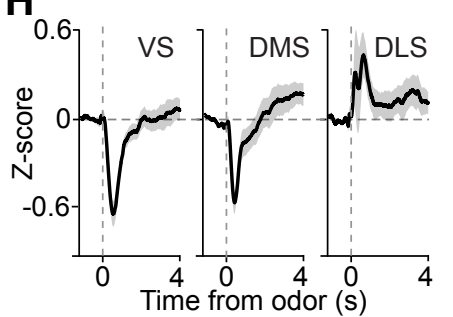

C

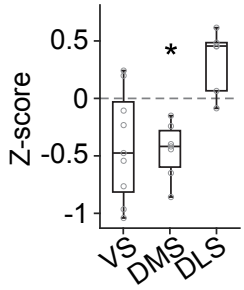

F

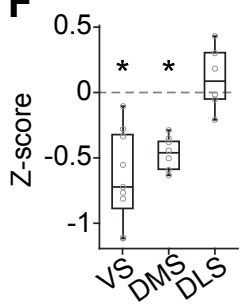

I

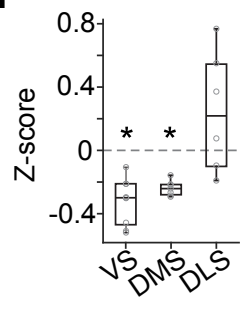


Figure 5. No inhibition by negative prediction error in dopamine axons in DLS (A) Activity pattern in each recording site aligned at small water. (B) Average activity pattern in each brain area (mean \pm SEM). (C) Mean responses to small water (0.3-1.3 s after water onset) were negative in VS and DMS ( $\mathrm{p}=0.031, \mathrm{p}=0.0025$, responses versus baseline), but not in DLS. The responses were different across striatal areas $(\mathrm{p}=0.0013$, ANOVA; $\mathrm{p}=0.0042$, VS versus DLS; $\mathrm{p}=2.8 \times 10^{-4}$, DMS versus DLS). (D) Activity pattern aligned at water timing in error trials. (E) Average activity pattern in each brain areas (mean \pm SEM). (F) Mean responses in error trials (0.3-1.3 s after water timing) were negative in VS and DMS $\left(p=6.2 \times 10^{-4}, p=6.5 \times 10^{-5}\right.$, responses versus baseline), but not in DLS. The responses were different across striatal areas $\left(\mathrm{p}=1.5 \times 10^{-4}\right.$, ANOVA; $p=5.8 \times 10^{-4}$, VS versus DLS; $p=1.6 \times 10^{-4}$, DMS versus DLS). (G) Activity pattern aligned at CS(-) in a fixed reward amount task. (H) Average activity pattern in each brain area (mean \pm SEM). (I) Mean responses at CS(-) (-1-0 s before odor port out) were negative in VS and DMS ( $\mathrm{p}=1.4 \times 10^{-4}, \mathrm{VS} ; \mathrm{p}=1.0 \times 10^{-5}, \mathrm{DMS}$, responses versus baseline), but not in DLS. Responses were different across striatal areas $\left(p=2.5 \times 10^{-4}\right.$, ANOVA; $p=0.0012$, VS versus DLS; $\mathrm{p}=0.0065$, DMS versus DLS). 
Figure 6

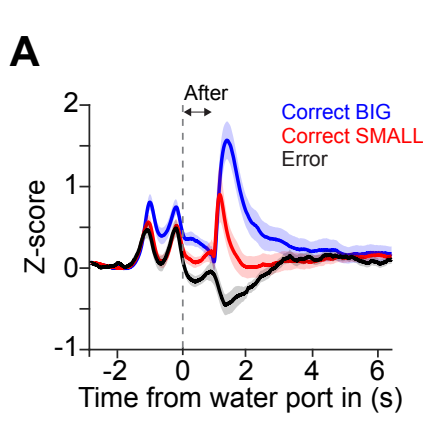

D Odor BIG - Odor SMALL

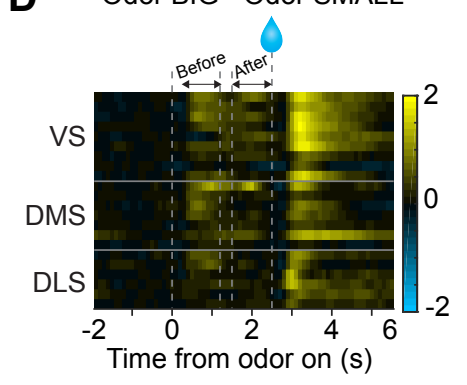

E

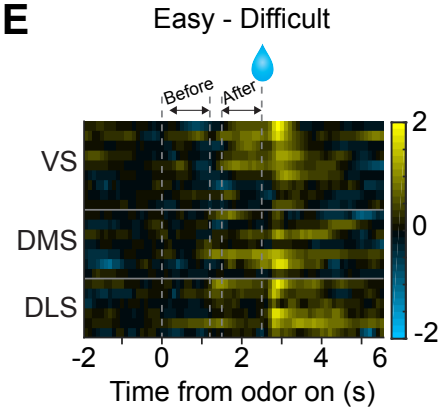

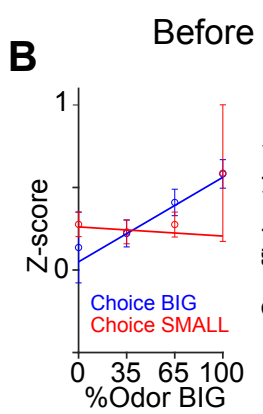

Odor BIG - Odor SMALL
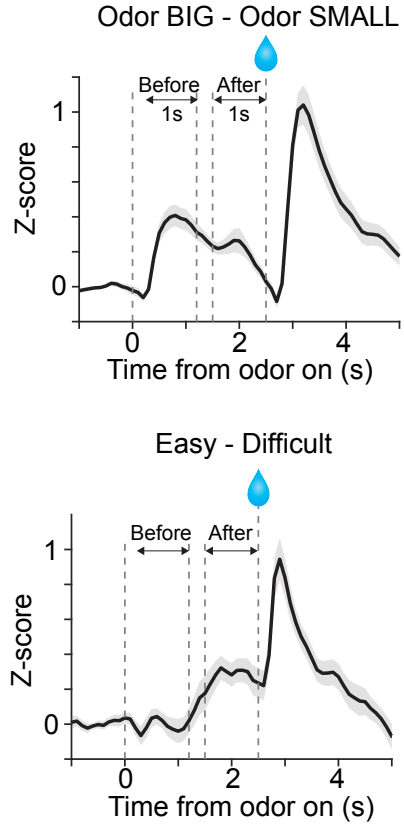

C

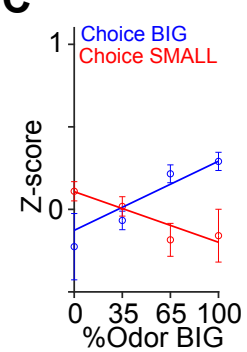

After choice

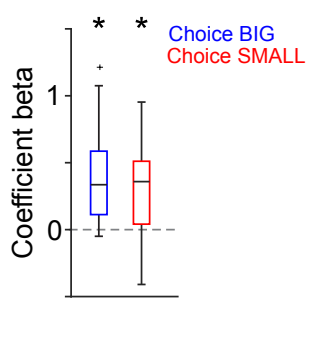

F Odor BIG - Odor SMALL
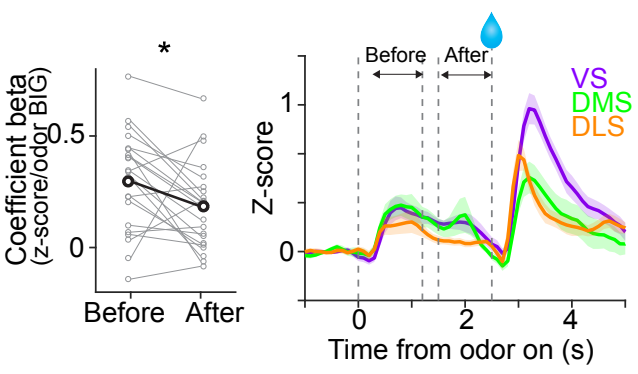

G
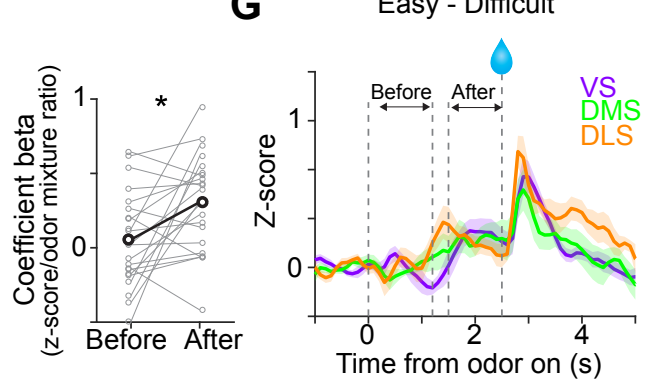
Figure 6. Dopamine signals stimulus-associated value and sensory evidence with different dynamics (A) Dopamine axon activity pattern aligned to time of water port entry for all animals (mean $\pm \mathrm{SEM}$ ). (B) Responses before choice (-1-0 s before odor port out) were fitted with linear regression with odor mixture ratio, and coefficient beta (slope) for all the animals are plotted. Correlation slopes were significantly positive for choice of the BIG side $\left(\mathrm{p}=5.6 \times 10^{-6}\right)$, but not significant for choice of the SMALL side $(\mathrm{p}=0.42)$. (C) Responses after choice $(0-1 \mathrm{~s}$ after water port in) were fitted with linear regression with stimulus evidence (odor \%) and coefficient beta (slope) for all the animals are plotted. Correlation slopes were significantly positive for both choice of the BIG side $\left(p=1.4 \times 10^{-5}\right)$ and of the SMALL side $\left(p=2.2 \times 10^{-4}\right)$. (D) Dopamine axon activity with an odor that instructed to choose BIG side (pure odor, correct choice) minus activity with odor that instructed to choose SMALL side (pure odor, correct choice) in each recording site (left), and the average difference in activity was plotted (mean $\pm \mathrm{SEM}$, middle). Correlation slopes between responses and stimulus-associated value (water amounts) significantly decreased after choice ( $\mathrm{p}=0.025$, before choice ( $-1-0 \mathrm{~s}$ before odor port out) versus after choice $(0-1 \mathrm{~s}$ after water port in), pure odor, correct choice). (E) Dopamine axon activity when an animal chose SMALL side in easy trials (pure odor, correct choice) minus activity in difficult trials (mixture odor, wrong choice) in each recording site (left), and the average difference in activity was plotted (mean \pm SEM, center). Coefficient beta between responses to odors and sensory evidence (odor \%) significantly increased after choice ( $\mathrm{p}=0.0078$, before choice versus after choice). (F) Average difference in activity (odor BIG minus odor SMALL) before and after choice in each striatal area. The difference of coefficient (before versus after choice) was not significantly different across areas ( $\mathrm{p}=0.86$, ANOVA). (G) Average difference in activity (easy minus difficult) in each striatal area. The difference of coefficient (before versus after choice) was not significantly different across areas $(\mathrm{p}=0.25$, ANOVA). 


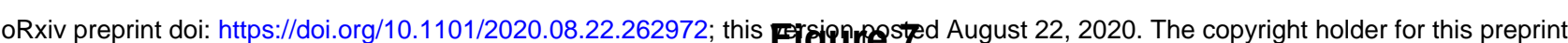
(which was not certified by peer review) is the author/funder, who has gralled bohxiv a license to display the preprint in perpetuity. It is made available under aCC-BY-NC-ND 4.0 International license. B

Adding perceptual noise
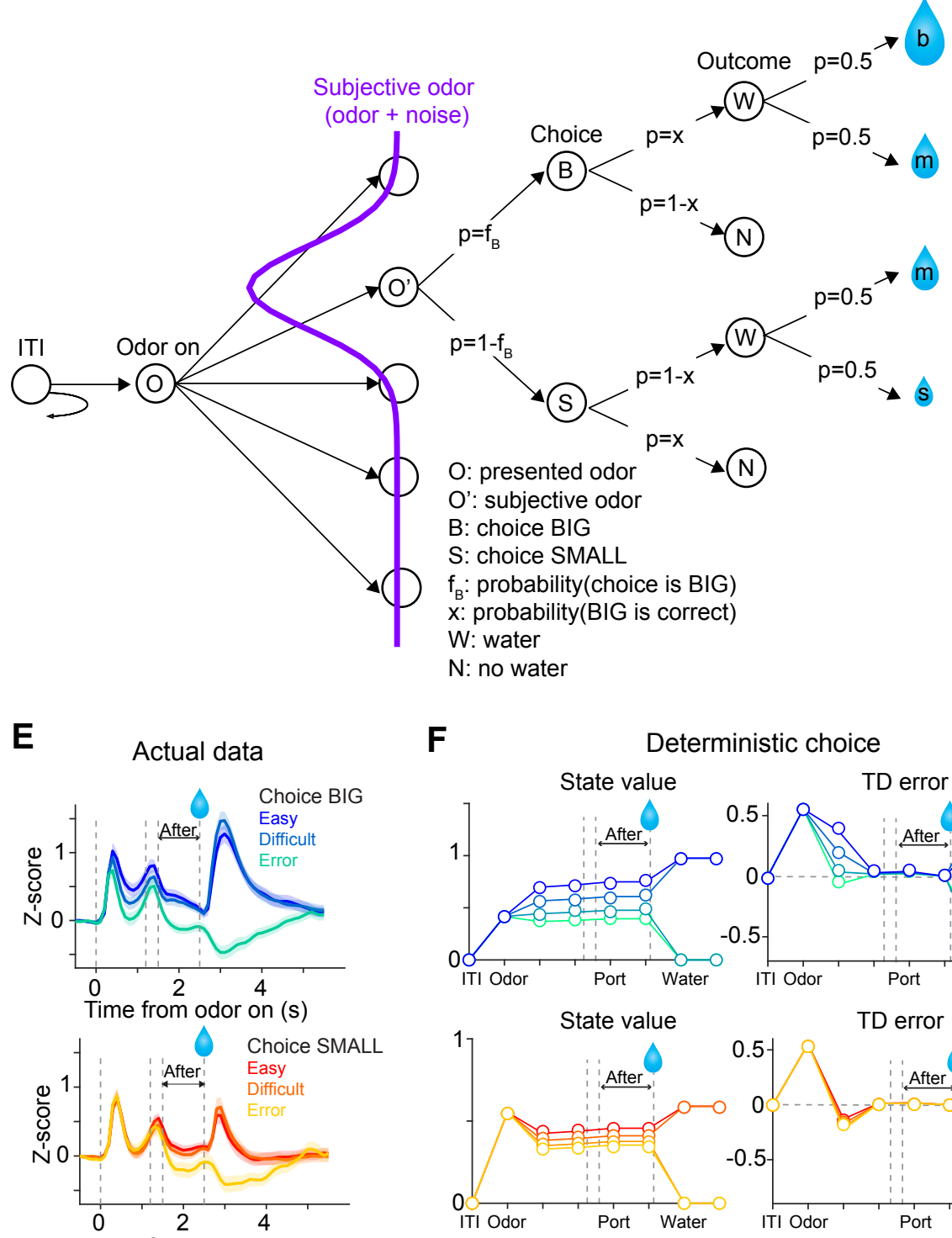

Time from odor on (s)

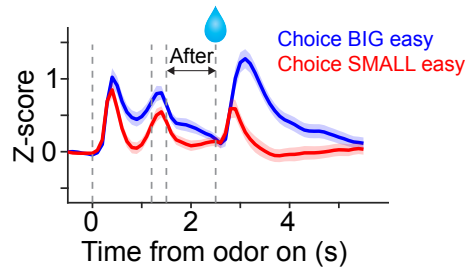

$\mathbf{F}$

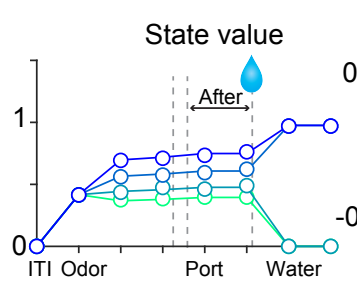

Deterministic choice
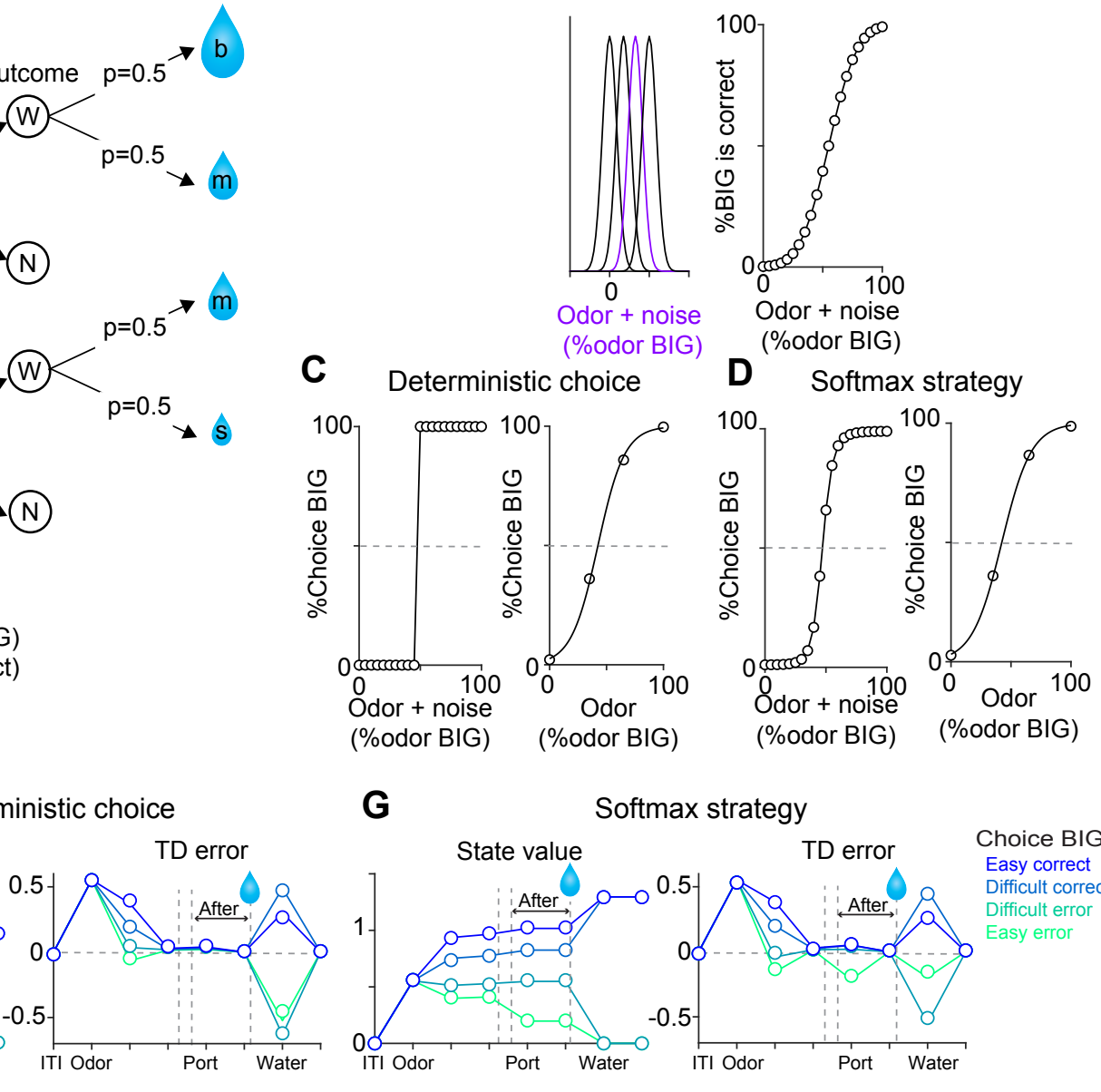
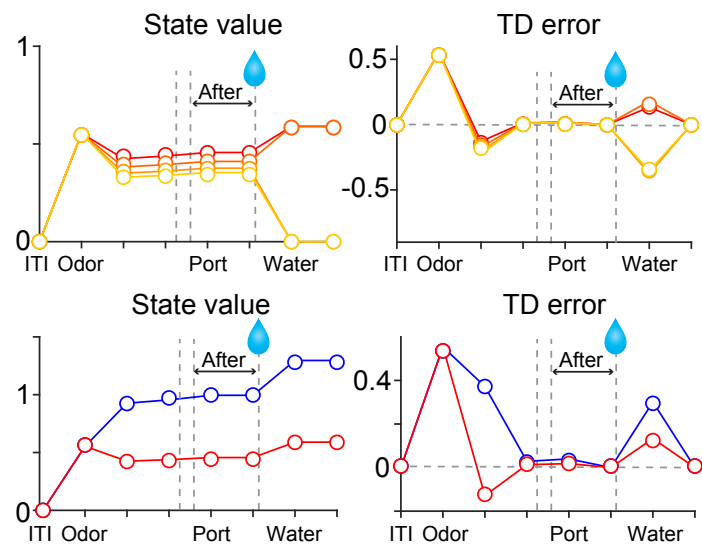

G

Softmax strategy
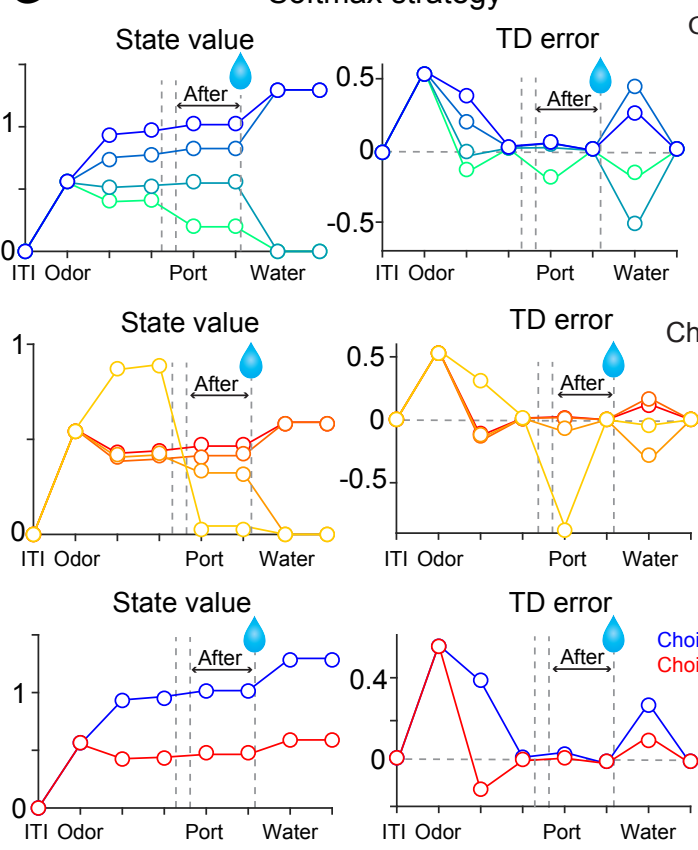

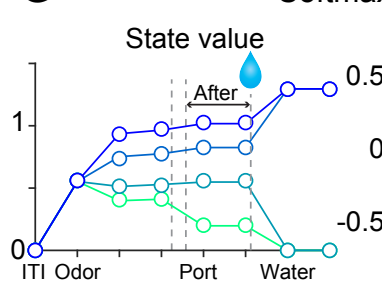

Choice BIG

Easy correct

Difficult correct
$\mathbf{E}$

\section{$H$}

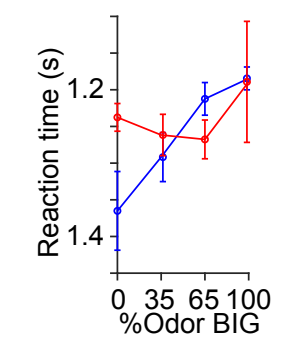

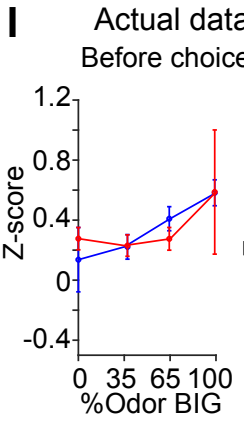

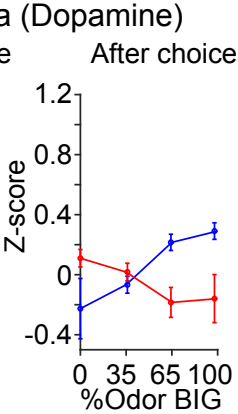

J Model (deterministic choice)
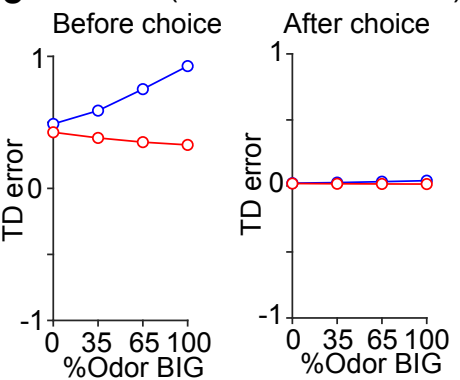

K Model (Softmax strategy)
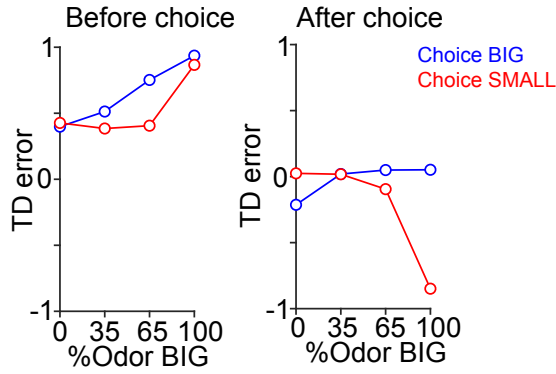


\section{Figure 7. TD error dynamics capture emergence of sensory evidence after stimulus-} associated value in dopamine axon activity (A) Trial structure in the model. Some repeated states are omitted for clarification. (B-D) Models were constructed by adding perceptual noise with normal distribution to each experimenter's odor (B left, subjective odor), calculating correct choice for each subjective odor (B right), and determining choice for each subjective odor (C or $\mathrm{D}$ left) according to choice strategy in the model. The final choice for each objective odor by experimenters (odor \%) was calculated as the weighted sum of choice for subjective odors (C or D right). (E) Dopamine axon activity in trials with different levels of stimulus evidence: easy (pure odor, correct choice), difficult (mixture odor, correct choice), and error (mixture odor, error), when animals chose the BIG side (top) and when animals chose the SMALL side (middle). Bottom, dopamine axon activity when animals chose the BIG or SMALL side in easy trials (pure odor, correct choice). (F, G) Time-course in each trial of value (left) and TD error (right) of a model. $(\mathrm{H})$ Line plots of actual reaction time from Figure 1G. Y-axis are flipped for better comparison with models. (I) Line plots of actual dopamine axon responses before and after choice from Figures 6B and 6C. $(\mathrm{J}, \mathrm{K})$ Model responses before and after choice were plotted with sensory evidence (odor \%). 

available under aCC-BY-NC-ND 4.0 International license.

\section{Figure $\mathbf{S 1}$}

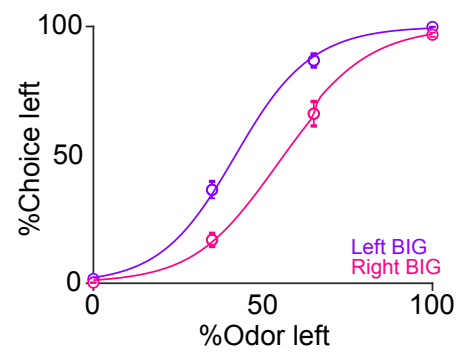

\section{Figure S1. Average psychometric curve in odor manipulation blocks}

$\%$ of choice of a left port when a left port is the BIG side or when a right port is the BIG side (mean \pm SEM) and the average psychometric curve for each case. 
Figure S2

A

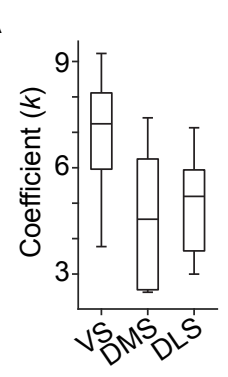

B

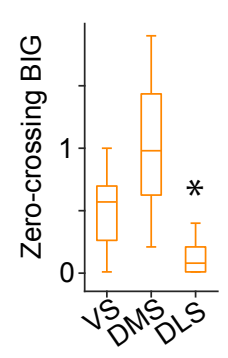

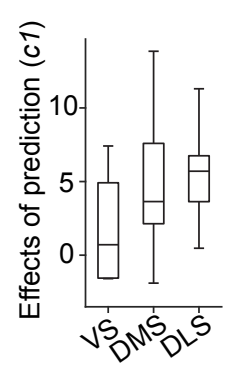

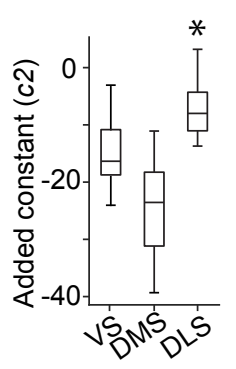

C

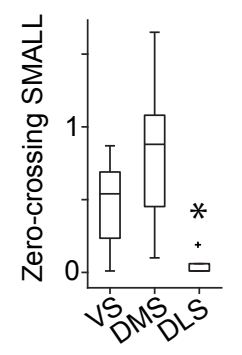

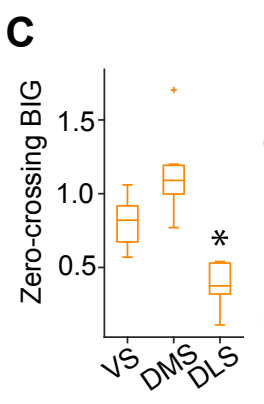

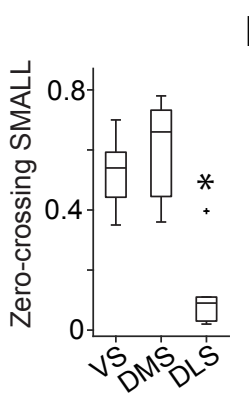

D
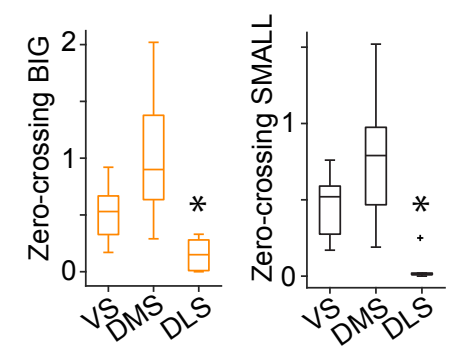

Figure S2. Zero-crossing points across the striatum with different methods (A) Each regression coefficient in the response function shown in Figure 4C. Fitting was performed by response $=k\left(\mathrm{R}^{\alpha}+c 1 \times \mathrm{S}+c 2\right)$, where $\mathrm{R}$ is the water amount, $\mathrm{S}$ is SMALL side (see Methods). (B) Zero-crossing points with linear function ( $p=0.003$ for $B I G ; p=6.1 \times 10^{-4}$ for SMALL, ANOVA). (C) Zero-crossing points with power function using a before-water time window ( -1 to $-0.2 \mathrm{~s}$ before water) as baseline. $\left(p=5.8 \times 10^{-5}\right.$ for $B I G ; p=2.1 \times 10^{-4}$ for SMALL, ANOVA). (D) Zero-crossing points using kernel models with power function $\left(p=0.0033\right.$ and $p=8.9 \times 10^{-4}$, ANOVA $)$. 
Figure S3

A

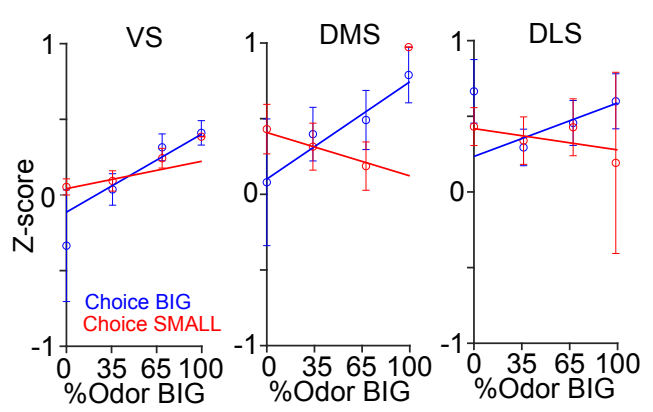

B

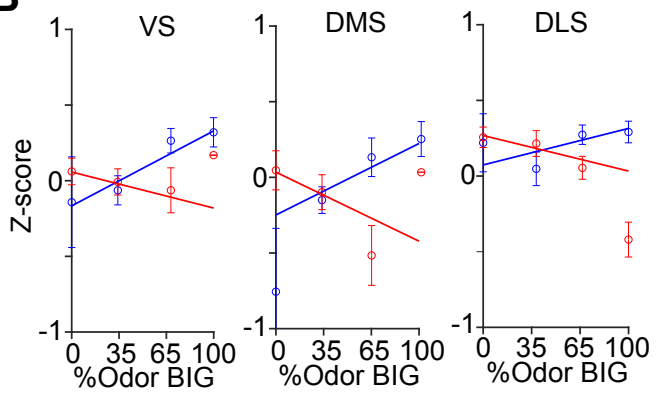

Figure S3. Dopamine axon responses before and after choice in each striatal area (A) Responses before choice (-1-0 s before odor port out) was fitted with linear regression with sensory evidence (odor \%) and average fitted lines in each striatal area were plotted. The correlation slope for small choice was slightly modulated by striatal areas ( $\mathrm{p}=0.0043$, ANOVA; $\mathrm{p}=0.0013$, VS versus DMS). (B) Responses after choice (0-1 s after water port in) was fitted with linear regression with sensory evidence and an average fitted line of each striatal area was plotted. The correlation slope was not significantly modulated by striatal areas $(\mathrm{p}=0.35$ for choice BIG; $\mathrm{p}=0.35$ for choice SMALL, ANOVA). 
A

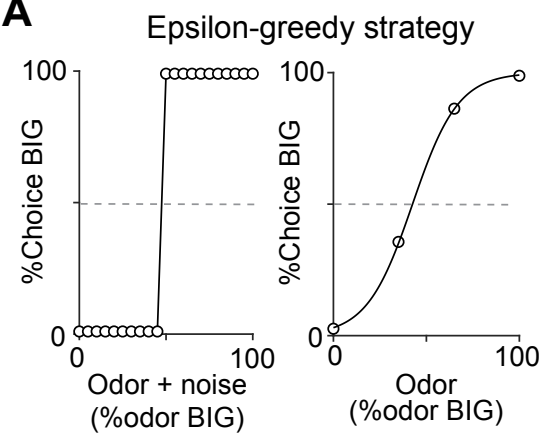

B

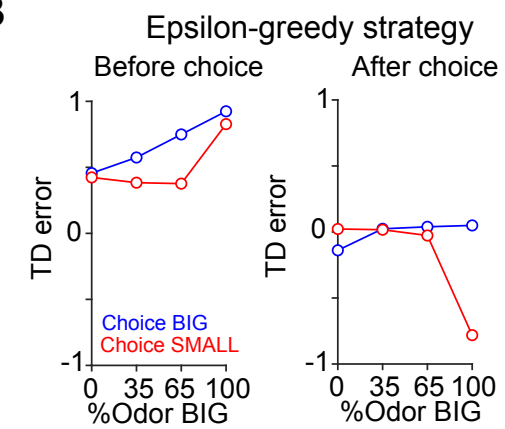

Matching strategy

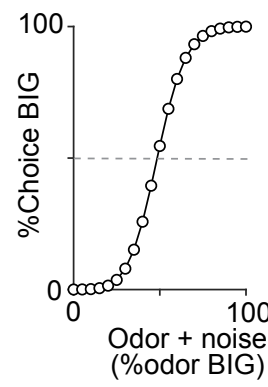

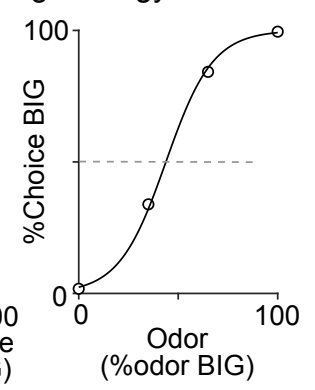

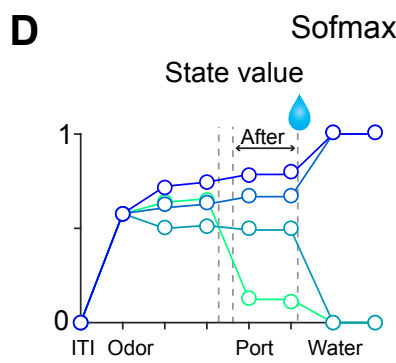

Sofmax (equal water)

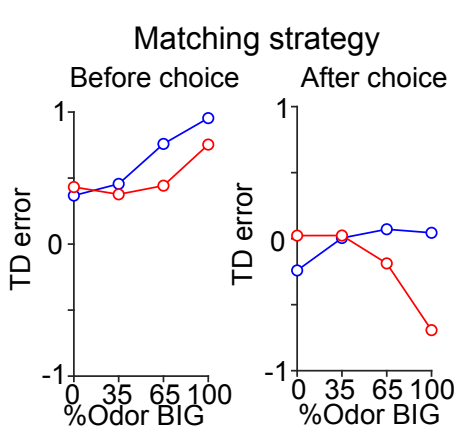

$\mathbf{E}$

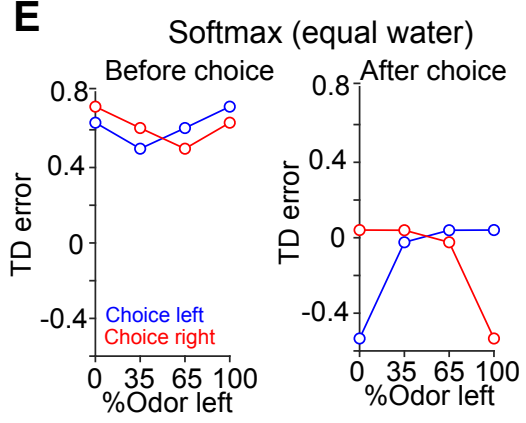

C
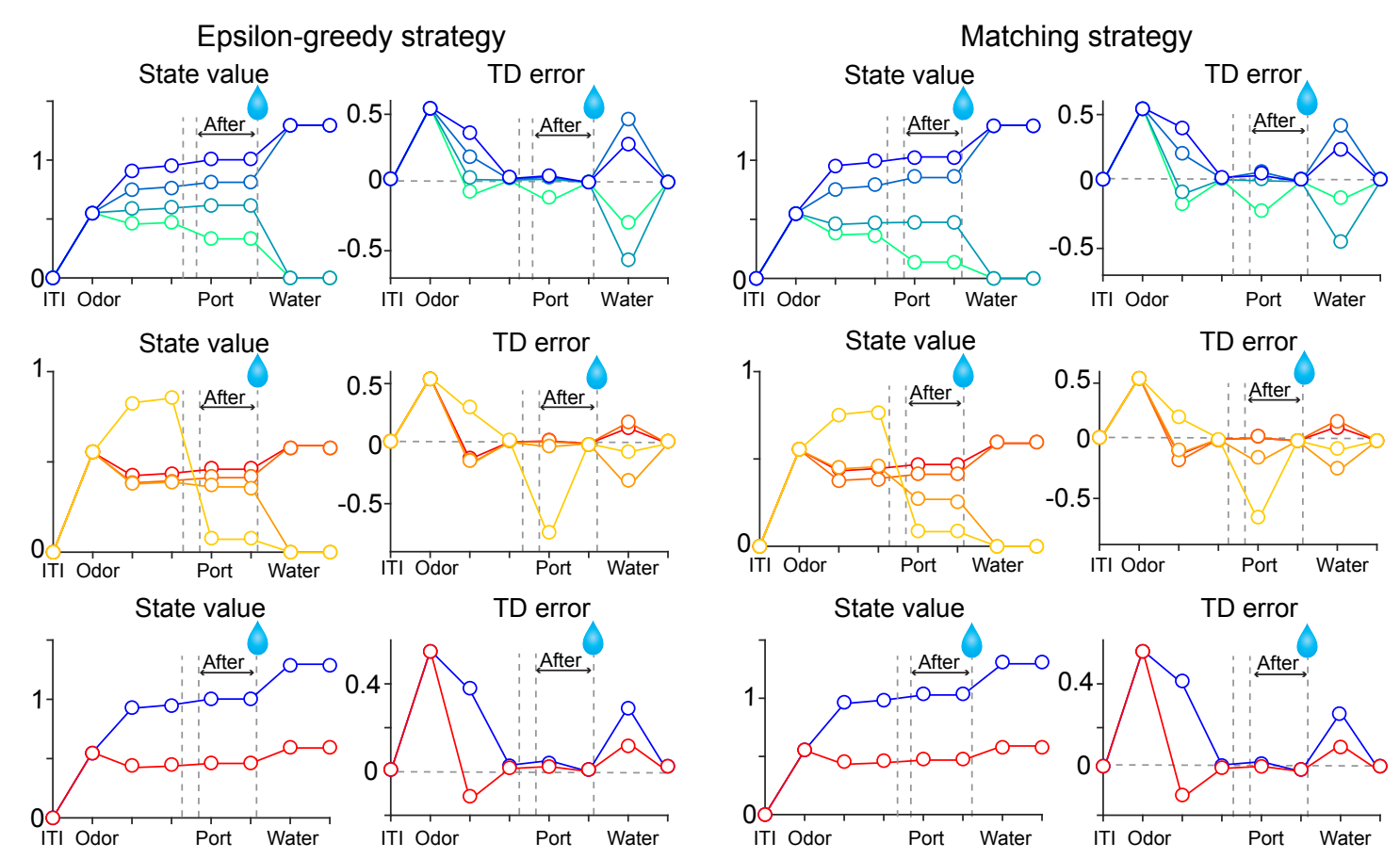

Choice SMALL

Easy correct

Difficult correct

Difficult error

Easy error
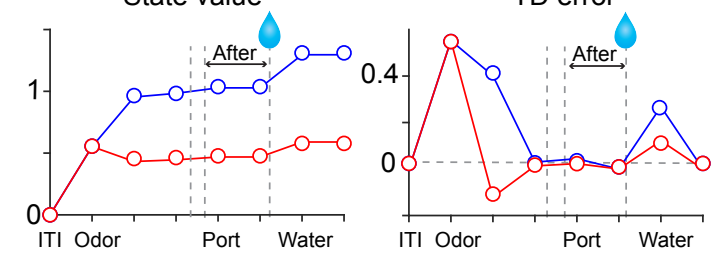

Choice BIG easy Choice SMALL easy

Figure S4. TD errors with stochastic choice strategies. (A) choice for each subjective odor (left) and choice for each objective odor (right) with epsilon greedy strategy and matching strategy. (B) TD errors with different sensory evidence (odor \%) before and after choice in each model. (C) The temporal dynamics of state values and TD errors in each model. (D) The temporal dynamics of state values and TD errors with a softmax choice strategy (Figure 7D) but with equal amounts of water for both water ports. (E) TD errors with different levels of sensory evidence (odor \%) before and after choice in model from $\mathrm{D}$. 